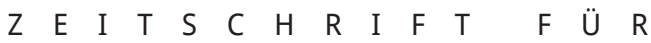

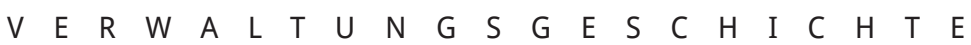

B A N D 1,20016

S E I T E $54-7 \quad 7$

D O I : $10.2478 / A D H I-2018-0004$

\title{
Verwaltungsrechtswissenschaft und Geschichtsschreibung
}

\author{
Narrative über Vergangenes - Versuch einer Typologie
}

BENJAMIN SCHINDLER ${ }^{1}$

\section{Einleitung}

Die Verwaltungsrechtswissenschaft beschäftigt sich in erster Linie mit dem geltenden Recht und seiner Anwendungspraxis in Verwaltung und Justiz. Die Auseinandersetzung mit der Vergangenheit steht nicht im Zentrum des Interesses - weder in der Forschung, noch in der Lehre. Rechtshistorikerinnen und -historiker wiederum beschäftigen sich traditionell mit dem Römischen Recht, dem Zivilrecht, dem Strafrecht oder dem Verfassungsrecht. ${ }^{2}$ Diese fachliche Fokussierung wird zementiert durch eine entsprechende Ausgestaltung der universitären Curricula und Umschreibung von Lehrstuhlprofilen. Dennoch hat sich die Wissenschaft vom Verwaltungsrecht immer wieder mit der Geschichte der Verwaltung und des Verwaltungsrechts, aber auch mit der Vergangenheit der eigenen Disziplin befasst. Eine explizite Selbstreflexion über historische Forschung - also eine Meta-Theorie - fehlt in der Verwaltungsrechtslehre weitestgehend, dies im Gegensatz zur Geschichtswissenschaft und zur Rechtsgeschichte. ${ }^{3}$ Der Grund hierfür mag sein, dass die praxis- und gegenwartsbezogene Verwaltungsrechtswissenschaft insgesamt weniger einem Rechtfertigungsdruck ausgesetzt ist als eine Disziplin, welche sich ausschließlich mit der Vergangenheit beschäftigt. Oft erinnern die knappen historischen Einleitungen in den Verwaltungsrechts- lehrbüchern denn auch eher an dekorative Ornamente bildungsbürgerlicher Wissenskultur. ${ }^{4}$ Es sind keine raumfüllenden und vertieften Auseinandersetzungen mit dem Gegenstand Geschichte. Ihre Verfasser sind zudem keine Historiker und meist nicht einmal Rechtshistoriker. Elaborate solcher "Amateurhistoriker ${ }^{5}$ stehen damit unter dem Generalverdacht, wenig bis nichts zur wissenschaftlichen Erkenntnis beizutragen.

Dieser Beitrag möchte anhand verschiedener Beispiele aufzeigen, dass die Verwaltungsrechtslehre in der Geschichte durchaus mehr sah (und sieht) als bloße Ornamentik. Wissenschaftliche Erkenntnis kann ein Grund sein. Vielfach geht es jedoch (auch) darum, gegenwartsbezogene wissenschaftliche Aussagen in eine Erzählung einzubetten und sich selber als Teil einer positiv oder negativ konnotierten Entwicklungsgeschichte zu positionieren. Eine geschichtliche Darstellung kann schließlich als `Argumentationspool dienen und für das heute geltende Recht instrumentalisiert werden. ${ }^{6}$ Diese verschiedenen Erzähltraditionen sind Teil der Geschichte der Verwaltungsrechtslehre als wissenschaftliche Disziplin. Sie sollen im Sinne einer Meta-Geschichte herausgearbeitet und transparent gemacht werden. ${ }^{7}$ Es soll aber auch den Gründen nachgespürt werden, weshalb die Geschichte von gewissen Autoren ganz ausge- 
blendet wird. Auch wenn Narrative über die Vergangenheit Konjunkturen und Moden ausgesetzt sind, folgt die Darstellung nicht einer chronologischen Einteilung in Epochen, sondern zielt auf eine Typologie historiografischer Stile. ${ }^{8}$ Sie soll helfen, die unterschiedlichen Erzählstränge im Verwaltungsrecht transparent zu machen, und damit einen Beitrag zur wissenschaftlichen Selbstreflexion leisten. Wie haben die Vertreter der Verwaltungsrechtswissenschaft Geschichte geschrieben und warum haben sie es so getan? Quellenbasis dieser Typologie sind in erster Linie die >Klassiker«, das heißt die anerkannten Standardwerke der Verwaltungsrechtslehre. Sie repräsentieren den wissenschaftlichen 'Mainstream und haben auf ganze Generationen von Wissenschaftlern, aber auch Richtern und Anwälten prägend gewirkt. Der Beobachtungszeitraum erstreckt sich vom ausgehenden 19. Jahrhundert - als sich das Verwaltungsrecht als eigenständige Wissenschaftsdisziplin im heutigen Sinn etablierte - bis in die Gegenwart. ${ }^{9}$

\section{Geschichte als Baum der Erkenntnis}

\section{Juristische Methodik und historische Auslegung}

Es entspricht kontinentaleuropäischem Rechtsverständnis, dass das Handeln von Verwaltung und Justiz seine zentralen Impulse aus dem geschriebenen Recht erhält: Normen des Völkerrechts, des Verfassungsrechts, des Gesetzesrechts und des Verordnungsrechts. Ausdruck findet dieses Denken im Legalitätsprinzip oder dem Grundsatz der Gesetzmäßigkeit. Die Auslegung (Exegese) von Rechtsquellen mit autoritativem Geltungsanspruch steht daher im Zentrum juristischer Tätigkeit; Rechtswissenschaft ist eine hermeneutische Disziplin. Das Verstehen des Textes ist Voraussetzung dafür, dass er durch die Verwaltung oder durch Gerichte auf einen konkreten Sachverhalt angewendet werden kann (sog. Subsumtion). ${ }^{10}$ Zum Kanon der anerkannten Auslegungsmethoden gehört auch die historische Auslegung. Während die "subjektiv-historische Methode» den Willen des historischen Gesetzgebers zu ergründen versucht, zielt die "objektiv-historische Methode" darauf ab, eine Norm aus dem allgemeinen zeithistori- der alten Streitfrage, inwiefern der vermeintliche oder tatsächliche "Wille des Gesetzgebers « für die heutige Rechtsanwendung maßgebend sein soll, ist die Befassung mit der Vergangenheit oft notwendig, um das geltende Recht zu verstehen. Etwa, weil ein Normtext Worte verwendet, die im heutigen Sprachgebrauch unüblich geworden sind oder anders gebraucht werden. ${ }^{12}$ Gerade im Verwaltungsrecht sind zudem zahlreiche Normen anzutreffen, welche als Reaktion auf bestimmte gesellschaftliche Herausforderungen erlassen wurden und ohne das entsprechende Kontextwissen nicht verstanden werden können. Die Befassung mit der Vergangenheit, insbesondere mit den "Materialien« der Gesetzgebung, gehört somit zum unverzichtbaren juristischen "Handwerkszeug ${ }^{13}{ }^{13}$ Diese Auseinandersetzung mit der Geschichte erfolgt immer gegenwartsbezogen - eben nur, soweit sie für eine konkrete Rechtsfrage von heute relevant ist - und meist stark fokussiert auf einen engen Kanon etablierter juristischer Quellen, wie den Berichten und Anträgen der Regierung an das Parlament oder den Protokollen der parlamentarischen Debatte.

\section{Historisches Wissen als Erkenntnisquelle}

Losgelöst von diesem sehr fokussierten und engen historischen Interesse der juristischen Auslegungsmethodik haben die Protagonisten der Verwaltungsrechtslehre im deutschsprachigen Raum großen Wert auf die Geschichte als Erkenntnisquelle gelegt. Otto Mayer (1846-1924) veröffentlichte in den Jahren 1895 und 1896 erstmals sein Deutsches Verwaltungsrecht. Mit seinem Versuch, das gesamte obrigkeitliche Handeln des Staates juristisch-dogmatisch zu erfassen, wird dieses Werk auch als das "Gründungsbuch des Verwaltungsrechts" im deutschsprachigen Raum bezeichnet. ${ }^{14}$ Nach einer Einleitung stellte Mayer seinem Werk einen drei Paragrafen umfassenden ersten Abschnitt voran, in welchem "Die geschichtlichen Entwicklungsstufen des deutschen Verwaltungsrechts « dargestellt wurden..$^{15} \mathrm{Zu}$ Beginn des Abschnitts rechtfertigte Mayer, weshalb die Darstellung der Geschichte der Verwaltung und des Verwaltungsrechts vergleichsweise viel Raum einnehme:

"Das heutige Verwaltungsrecht hat hinter sich seine Geschichte wie jede Rechtsart. Die rechte Erkenntnis seiner Vorstufen ist aber von einer ganz unvergleich- 
lichen Wichtigkeit. Wir müssen uns nur gegenwärtig halten, wie merkwürdig für die geschichtliche Betrachtungsweise der Punkt ist, auf welchem wir heute stehen. Wir können von hier aus zurückblicken auf mehrere ungemein starke und tiefgehende Umwälzungen, die sich in verhältnismäßig kurzem Zeitraum vollzogen und soeben erst ihren Abschluß gefunden haben. [...] Diesem raschen Gange entspricht der Zustand, der uns vor Augen liegt. Das wirkliche Recht ist noch erfüllt mit Trümmern vorausgehender Entwicklungsstufen, die als Widerspruch mit dem Grundgedanken des neuen Rechts dastehen und allmählich verschwinden oder sich umbilden müssen. Die Wissenschaft ihrerseits, von der Praxis zu schweigen, ist zum Teil noch gebunden in älteren Anschauungen, die zum neuen Rechte nicht mehr passen, und hängt an Ausdrucksweisen, welche heute nur in gänzlich verändertem Sinne zu gebrauchen sind. Wer sich hier zurechtfinden soll, für den ist die erste Bedingung, daß er der damit gegebenen Gegensätze sich immer klar bewußt bleibt. ${ }^{16}$

Auch Fritz Fleiner (1867-1937) widmete in seinen populären und 1911 erstmals erschienenen Institutionen des Deutschen Verwaltungsrechts der "Geschichtlichen Entwicklung des deutschen Verwaltungsrechts und der Verwaltungsrechtswissenschaft « einen eigenen Paragrafen. ${ }^{17}$ Auch er rechtfertigte seine Ausführungen zur Vergangenheit:

"Nicht aus einer gewaltigen Krise von Staat und Gesellschaft ist das deutsche Verwaltungsrecht der Gegenwart hervorgegangen, sondern aus einem langsamen Umbildungsprozesse in den einzelnen Territorien. In keinem einheitlichen Tempo sind diese Bewegungen in den einzelnen deutschen Ländern vor sich gegangen, und nirgends hat das jeweilige neue Recht das alte vollkommen beseitigt; in jede Periode ragen rechtliche Überreste überwundener Anschauungen herein. Aber in einer jeden Periode bestimmte eine neue, von den früheren Bestrebungen abweichende Auffassung die Stellung der öffentlichen Gewalt zum Untertan und zum Recht. Dabei muß man sich stets gegenwärtig halten, daß sich von Epoche zu Epoche die Anschauungen über den Umfang der öffentlichen
Mayer und Fleiner machten in ihren Texten deutlich, dass "die rechte Erkenntnis seiner Vorstufen« zentral sei, um das heutige Verwaltungsrecht $\mathrm{zu}$ verstehen. Fleiner erwähnte in anderem Kontext, dass "nicht selten die Entwicklung der Rechtsinstitutionen stärker zu uns [spricht], als das Gewordene ${ }^{19}$. Mayer wie Fleiner verwiesen zudem auf die "Trümmer" beziehungsweise die "Überreste« der Vergangenheit, welche Zeugen "vorausgehender Entwicklungsstufen « und »überwundener Anschauungen" seien und welche es zu überwinden gelte. Damit wird deutlich, dass es beiden Autoren nicht nur um das Verstehen und Erklären ging, sondern mit dieser Erkenntnis auch rechtspolitische Forderungen verknüpft wurden. Zentrales Anliegen war es, das Konzept des bürgerlich-liberalen »Rechtsstaats « konsequent umzusetzen und "polizeistaatliche« Überbleibsel aus dem Recht zu verbannen.

Trotz vieler Gemeinsamkeiten machen die Zitate deutlich, dass Mayer und Fleiner von einem unterschiedlichen Geschichtsverständnis geprägt waren. Während Mayer die "ungemein starke[n] und tiefgehende[n] Umwälzungen " in den Vordergrund rückte, interpretierte Fleiner die Geschichte eher im Sinne eines kontinuierlichen und evolutiven "Umbildungsprozesses «. ${ }^{20}$ Die unterschiedliche Gewichtung von Brüchen und Kontinuitätslinien hat mehrere Gründe. Einer dürfte der biografisch-geografische Hintergrund sein. Fleiner wie Mayer entstammten zwar beide einem ähnlichen Milieu: protestantisch, bürgerlich, wohlhabend und liberal. Der 1846 im fränkischen Fürth geborene Otto Mayer erlebte den Deutsch-Französischen Krieg (1870-1871) und die Gründungsphase des Deutschen Kaiserreichs (1871) intensiv. Er war seit 1872 in Mülhausen und seit 1880 in Straßburg tätig, also in einem Gebiet, das als Teil von Elsass-Lothringen von Frankreich zum Deutschen Reich kam. ${ }^{21}$ Mayers Erfahrungen mit der öffentlichen Verwaltung waren diejenigen mit einem bürokratisch und monarchisch geprägten Beamtentum. Fleiner wurde demgegenüber 1867 im schweizerischen Aarau geboren - in eine Zeit, in der sich der schweizerische Bundesstaat (gegründet 1848) stabilisiert hatte und keine grundlegenden politischen Umbrüche mehr erfolgten. ${ }^{22}$ Das politische und gesellschaftliche Umfeld war klar bürgerlich-liberal dominiert. Fleiners Urgroßsvater mütterlicherseits, der aus Deutschland stammende Heinrich Zschokke (1771-1848), war als Pädagoge, Zei- 
tungsverleger und Politiker ein Vorkämpfer des Liberalismus gewesen und fand auch in Deutschland Leser - übrigens auch im Elternhaus Otto Mayers. ${ }^{23}$ Zschokke verfasste das 1822 erstmals erschienene Werk Des Schweizerlands Geschichte für das Schweizervolk. Dieses für breite Bevölkerungsschichten geschriebene Werk prägte das liberale Geschichtsbild der Schweiz für Generationen. Zschokke untermalte seine Geschichte mit Freiheitslegenden und sah die Aufgabe künftiger Generationen darin, sich der Überlieferung verpflichtet $\mathrm{zu}$ fühlen und auch in Zukunft für die Freiheit einzustehen und Gegentendenzen im Keim zu ersticken. Im Vorwort verdeutlicht Zschokke seine volkserzieherische Intention mit einer biblischen Metapher:

"So merket denn auf meine Rede, ihr Alten und Jungen. Die Geschichte verflossener Zeiten ist ein Baum der Erkenntnis des Guten und Bösen. «"24

Vor seinem biografisch-geografischen Hintergrund sah Otto Mayer seine Aufgabe als Rechtswissenschaftler vor allem darin, "Befreiungsarbeit « für ein neues, besseres Recht zu leisten. ${ }^{25}$ Die Verwaltung musste durch das Verwaltungsrecht rechtsstaatlich gebändigt werden. In Mayers fortschrittsgläubigem Narrativ ist die Geschichte somit das Hinaufklettern und Überwinden von "Entwicklungsstufen«. Auf diese heils- oder entwicklungsgeschichtliche Perspektive wird später noch näher einzugehen sein (hinten, Abschnitt "Geschichte als Heilsgeschichte«). Im Gegensatz dazu musste Fleiner für die Schweiz nur noch begrenzt Pionierarbeit leisten. Das bürgerlich-liberale Gedankengut war etabliert, es galt lediglich die für das Verwaltungsrecht maßgeblichen Folgerungen zu ziehen. ${ }^{26}$ Anders als bei Mayer war die Geschichte somit weniger ein Erklimmen von Entwicklungsstufen als vielmehr ein kontinuierliches Werden und Vergehen. ${ }^{27}$ Staat und Verwaltung standen für ihn im Spannungsfeld zwischen Pflege der Tradition und Offenheit für neue Entwicklungen. ${ }^{28}$ Fleiner sah die Aufgabe der Wissenschaft darin, sich in diesem Spannungsfeld als Brückenbauer zu betätigen. Es galt, das >Gute` zu bewahren und das `Böse` absterben zu lassen. Auch wenn Fleiner die biblische Semantik vom Baum der Erkenntnis nicht verwendet, ist das Erbe seines Ahnen Zschokke doch deutlich spürbar:

\begin{abstract}
»Wenn wir dergestalt das Lebenskräftige von dem Absterbenden unterscheiden lernen, enthüllt sich unserm Blick das politisch Mögliche gegenüber der Utopie, und wir erleben Stunden, in denen wir eine Strecke Wegs in die verschleierte Ferne zu schauen vermögen und die Gegenwart uns erscheint als die Brücke zwischen Vergangenheit und Zukunft. Auf diese Weise machen wir die Vergangenheit dem Leben nutzbar. $\star^{29}$
\end{abstract}

\section{Fokussierung auf juristische Fragestellungen und Epochenbildung}

Wird die Befassung mit der Geschichte vor allem als Erkenntnis- oder Erklärungshilfe für das geltende Recht verstanden, führt dies zu einer klaren Fokussierung. Nicht die Geschichte der Verwaltung im Allgemeinen interessiert, sondern allein diejenigen Fragestellungen, welche auch die heutige Rechtswissenschaft umtreiben und prägen. ${ }^{30}$ Die bürgerlich-liberale Verwaltungsrechtslehre verstand das Verwaltungsrecht in erster Linie als Schutzwall der individuellen Freiheit gegenüber willkürlicher Behördenmacht. Der `Untertan` sollte nicht mehr bloß Gegenstand staatlicher Machtausübung sein, sondern Rechtssubjekt, welchem der Staat in "rechtlich geordneten und gebundenen Beziehungen gegenüber steht «. ${ }^{31}$ Aus diesem klaren Fokus ergab sich die aus Sicht der Verwaltungsrechtslehre naheliegende Epocheneinteilung. Ein sSchüler Otto Mayers, Walter Jellinek (1885-1955), ${ }^{32}$ rechtfertigte diesen Fokus in seinem 1927 erstmals erschienenen Lehrbuch zum Verwaltungsrecht und machte auch deutlich, inwiefern sich das Interesse des Juristen an der Vergangenheit von demjenigen des Historikers unterscheidet:

"Da Gegenstand des Verwaltungsrechts hauptsächlich die Beziehungen des einzelnen zum übergeordneten Staate sind, muß auch eine Geschichte des Verwaltungsrechts der Entwicklung dieser Beziehungen gewidmet sein. Die Geschichte der Behördenverfassung der aktiven Verwaltung tritt daneben an Bedeutung zurück, auch fehlt ihr das besondere juristische Element; ihre Darstellung gehört daher mehr zur allgemeinen Geschichte. Für die Beziehungen des einzelnen zum Staate aber lassen sich in der Neuzeit drei typische Regelungen feststellen: Die Regelung 
des älteren Justizstaates, des Polizeistaates und des heutigen Rechtsstaates. «"

Nach 1945 knüpfte die Rechtfertigung historischer Ausführungen an der älteren Literatur an, soweit sich die Literatur überhaupt noch mit der Vergangenheit befasste. ${ }^{34}$ Ernst Forsthoff (1902-1974) griff in seinem 1950 erstmals erschienenen Lehrbuch des Verwaltungsrechts die Argumente Mayers und Fleiners fast wörtlich auf und kam zum Schluss, dass "[d]as Verständnis der Verwaltung [...] also die Kenntnis ihrer Geschichte voraus[setzt]. «35 Dennoch distanzierte er sich - freilich nur in einer Fußnotenbemerkung - vom Dreigestirn Mayer, Fleiner und Jellinek, indem er die Epocheneinteilung in Justizstaat, Polizeistaat und Rechtsstaat verwarf. Forsthoff wollte den »mit dieser Dreiteilung leicht verbundenen Schematismus« vermeiden und hoffte, mit einer anderen Darstellung »dem berechtigten Anliegen der Verwaltungslehre und der Verwaltungssoziologie « besser zu entsprechen. ${ }^{36}$ In den Vordergrund rückte Forsthoff nun vor allem Aspekte der Verwaltungsorganisation und das Werden des Sozial- beziehungsweise Wohlfahrtsstaates. Dies vor dem Hintergrund einer stärkeren Betonung der Verwaltung als Leistungsträgerin und dem von Forsthoff propagierten Begriff der staatlichen "Daseinsvorsorge». Forsthoff distanzierte sich explizit von dem seines Erachtens zu einseitigliberal ausgerichteten Rechtsstaatsverständnis. ${ }^{37}$ Diese inhaltliche Neuausrichtung musste nun auch historisch anders untermauert werden. ${ }^{38}$

Die bisherigen Ausführungen machen deutlich, dass die Befassung der Verwaltungsrechtslehre mit der Vergangenheit nicht alleine von Erkenntnisinteressen getrieben war. Sie diente ganz wesentlich auch dazu, rechtspolitische Positionen in eine passende Erzählung einzubetten.

\section{Geschichte als Heilsgeschichte}

Otto Mayers Geschichtsbild steht exemplarisch für die Vorstellung, dass sich die Menschheit hin zum Höheren, Besseren entwickelt oder entwickeln soll: "Mit der Menschheit soll es höher hinauf; da kann es nicht in derselben Ebene weitergehen. «39 Dies zeigt sich schon
Deutschen Verwaltungsrecht: "Die geschichtlichen Entwicklungsstufen des deutschen Verwaltungsrechts«. Das Erklimmen dieser Stufen erfolgte für Mayer nicht naturgesetzlich von alleine, sondern war ein schmerzhafter Kampf. ${ }^{40}$ Ein Kampf, der sich vor allem gegen "unsere eigene Vergangenheit, die Rechtsanschauung des Polizeistaates «11 richtete. Das »Fortschleppen polizeistaatlicher Atavismen« bekämpfte Mayer »mit Scharfsinn und konstruktiver Folgerichtigkeit unablässig « ${ }^{42}$. Ziel dieses Kampfes und damit die zu erreichende Endstufe war für Mayer der Rechtsstaat. Darunter verstand Mayer den "Staat des wohlgeordneten Verwaltungsrechts «" und dies bedeutete "die tunlichste Justizförmigkeit der Verwaltung « ${ }^{44}$. Die Verwaltung sollte an das Gesetz gebunden sein, gegenüber den Individuen in der geordneten Form des Verwaltungsakts handeln und dabei der Kontrolle durch unabhängige Verwaltungsgerichte unterstehen. ${ }^{45}$ Hintergrund von Otto Mayers Geschichtsdarstellung waren neben seiner liberalen Grundhaltung der Entwicklungsgedanke des 19. Jahrhunderts und ein damit verbundener naturwissenschaftlicher Evolutionismus. ${ }^{46}$ Hinzu kommen bei Mayer sein starker christlicher Glaube und seine intensive Beschäftigung mit der Eschatologie. ${ }^{47}$ War der anzustrebende Endzustand des rechtsstaatlichen Verwaltungsrechts einmal erreicht, galt es ihn in der Logik Mayers auch zu bewahren. So findet sich im knappen Vorwort zur dritten Auflage des Deutschen Verwaltungsrechts von 1923 die zum geflügelten Wort gewordene Aussage: "Groß Neues ist ja seit 1914 und 1917 nicht nachzutragen. >Verfassungsrecht vergeht, Verwaltungsrecht besteht « «. ${ }^{48}$

Ganz anders das Vorwort zur Neuauflage von Fleiners Institutionen von 1928: "Die Neugestaltung des Verfassungsrechtes hat in Deutschland einen starken Einfluss auch auf das Verwaltungsrecht ausgeübt. [...] In der vorliegenden Auflage dieses Buches ist der Versuch unternommen worden, das Neue organisch mit den bewährten Grundsätzen des Deutschen Verwaltungsrechts $\mathrm{zu}$ verbinden.« Fleiner betonte damit in bewusstem Kontrast zu Mayer den kontinuierlichen Wandel und das Bedürfnis nach ständiger Erneuerung, welche aber auf dem Bewährten aufbauen soll. ${ }^{49}$ Dennoch ist auch Fleiners Verständnis der Verwaltungsrechtsgeschichte nicht völlig frei von heilsgeschichtlichen Elementen. So bezeichnete er die Schaffung einer unabhängigen Verwaltungsgerichtsbarkeit in metaphorischer Über- 
höhung als "Krönung des Rechtsstaates « ${ }^{50}$. Bei anderen Autoren wurde die Verwaltungsgerichtsbarkeit zum "Schlussstein im Gewölbe des Rechtsstaats «. ${ }^{51}$ Selbst bei Autoren, die auf historische Ausführungen weitgehend verzichteten, ist dieses heils- oder entwicklungsgeschichtliche Motiv zwischen den Zeilen herauszulesen. Zaccaria Giacometti (1893-1970) stellte in seinen 1960 erschienen Allgemeinen Lehren des rechtsstaatlichen Verwaltungsrechts ${ }^{52}$ fest, in der Schweiz befinde sich der Rechtsstaat mangels Ausbau der Verwaltungsgerichtsbarkeit "noch auf einer primitiveren Stufe«. Gleichzeitig prognostizierte er, dass sich »die Entwicklung selbstverständlich nach der rechtsstaatlichen Richtung “ bewegen werde. ${ }^{53}$

\section{Geschichte als Erzählung vom verlorenen Paradies}

Das Gegenstück zu einer heilsgeschichtlichen Betrachtung scheint prima vista der verklärende Blick zurück; die Erzählung von der sguten alten Zeit‘. Dass sich beide Perspektiven nicht ausschließen müssen, macht das Werk Fleiners deutlich. Er identifizierte die Vergangenheit nicht nur mit dem Polizeistaat, den es zu überwinden galt. Er betonte ebenso, dass das Neue auf dem Bewährten aufbauen und »organisch « weiterführen müsse. Bei Fleiner kam das Bedürfnis, sich in eine Tradition zu stellen und die Verbindung mit der Vergangenheit zu unterstreichen, bereits im "archaisierenden « ${ }^{54}$ Titel seines verwaltungsrechtlichen Hauptwerks zum Ausdruck. Die Institutionen des Deutschen Verwaltungsrechts knüpften an die Institutionen des römischen Rechts und des deutschen Privatrechts an. ${ }^{55}$ Und in einem Beitrag für die Festgabe zu Otto Mayers 70. Geburtstag verwies er darauf, dass es Aufgabe der Verwaltungsrechtswissenschaft sei, "einen Schatz nationalen, ungebrochenen Rechtsstoffs zu heben und geistig zu gestalten $\aleph^{56}$.

Betrachtet man das hohe Maß an Konstruktion und Abstraktion, mit welchem die Verwaltungsrechtswissenschaft gestaltend wirkte, erscheint diese Aussage allerdings eher dazu zu dienen, den Mangel an Tradition und Kontinuität einer noch jungen Wissenschaftsdisziplin rhetorisch klein zu reden. ${ }^{57}$ Zudem sollte die Dogmatik des Allgemeinen Verwaltungsrechts in einem Rechtsge- prägt war und bis heute ist, stabilisierend wirken. ${ }^{58} \mathrm{Im}$ Vorwort zu seinen Institutionen verwies Fleiner darauf, dass die Gesetze von Jahr zu Jahr komplizierter würden. Deshalb sei es die Aufgabe der Wissenschaft, die verwaltungsrechtlichen Erscheinungen auf ihre »juristischen Grundlinien zurückzuführen « ${ }^{59}$. Damit suggerierte Fleiner, diese Grundlinien seien schon dagewesen und es gelte lediglich sie für die Gegenwart offenzulegen.

Die heilsgeschichtliche Perspektive Otto Mayers musste schließlich $\mathrm{zu}$ dem Zeitpunkt in eine nostalgisch-verklärende Sichtweise umkippen, als der liberale Rechtsstaat in den 1930er-Jahren zunehmend unter Druck geriet. Walter Jellinek stellte in der 1931 erschienen Neuauflage seines Verwaltungsrechtslehrbuchs rhetorisch die Frage, ob »die Entwickelung [des Rechtsstaates] einen Ruhepunkt erreicht ${ }^{60}{ }^{0}$ habe. Darauf folgte ein Plädoyer für den Rechtsstaat, welches mit einem mythisch-verklärenden Blick in die Vergangenheit schließt:

\footnotetext{
"Trotz allem wird es in Deutschland beim Rechtsstaat bleiben. Ein Volk, das die Erzählung vom Michael Kohlhaas ersann, das die Rütliszene schuf, das die Antwort des Müllers von Sanssouci von Geschlecht zu Geschlecht überlieferte, das die richterliche Unabhängigkeit durch ein Martyrium erzwang, läßt sich sein Ideal weder durch ausländische Einflüsse noch durch vorübergehende Staatsnotwendigkeiten rauben. [...] Das Zeitalter des Rechtsstaates wird wohl für absehbare Zeit bestehen bleiben. « ${ }^{61}$
}

Die große Mehrzahl der Verwaltungsrechtswissenschaftler war seit dem ausgehenden 19. Jahrhundert einem liberalen Rechtsstaatsparadigma verpflichtet ${ }^{62}$ - zumindest, wenn man die Phase zwischen 1933 und 1945 und die ehemalige DDR ausblendet. Begleitet wurde diese Sichtweise von der Erzählung, dieser ideale Rechtsstaat sei im 19. Jahrhundert historische 'Realität gewesen. Während Jellinek und andere deutsche Autoren den Rechtsstaat in erster Linie mit einem gut funktionierenden Justizwesen identifizierten (Rechtsstaat als Gerichtsstaat), ${ }^{63}$ lag der Akzent in der Schweiz eher auf einem schlanken und unbürokratischen Staatswesen. Teil dieser Erzähltradition ist die >Beschreibung، einer Entwicklung, wonach der liberale Grundgedanke durch immer neue sozialstaatlich oder anderweitig »interventionistisch« motivierte Gesetze und Vorschriften unter 
Druck gerät. Dieser Unterton schwingt im erwähnten Vorwort zu Fleiners Institutionen mit und kommt auch andernorts in seinem Werk zum Ausdruck. ${ }^{64}$

In diesem Kontext ist auch die Verwendung des Begriffs des 'Nachtwächterstaats` durch die rechtswissenschaftliche Literatur zu lesen. Der durch Ferdinand Lassalle (1825-1864) geprägte politische Kampfbegriff verlor seine negative Konnotation und mutierte zur positiv wertenden Umschreibung einer vermeintlich empirisch nachgewiesenen historischen Begebenheit. ${ }^{65}$ Staatlicher Interventionismus und Überregulierung erscheinen so - wenn man naturwissenschaftliche Metaphern bemüht - als degenerative Erscheinungen. ${ }^{66}$ In einer eher theologisch geprägten Sprache erscheint diese Erzählung als Abgesang auf das verlorene Paradies. ${ }^{67}$ In einem stark kulturpessimistisch geprägten Aufsatz beschwor Hans Huber (1901-1987) 1971 den "Niedergang des Rechts und die Krise des Rechtsstaates ${ }^{68}{ }^{8}$. Mit einem an die biblische Sintflut erinnernden Sprachbild rief er künftige Juristengenerationen dazu auf, die Dämme zu festigen, wenn »in finsterer Nacht [...] im Sturm die Deiche zu brechen ${ }^{69}$ drohten.

\section{Geschichte als Purgatorium}

Zeitgleich mit Walter Jellineks Verwaltungsrecht erschien 1927 das Allgemeine Verwaltungsrecht des Österreichers Adolf Merkl (1890-1970). Der Vertreter der Wiener Rechtstheoretischen Schule verweigerte sich im Gegensatz zu Jellinek einem historisierenden Narrativ. Vielmehr benutzte Merkl die Geschichte gezielt als Mittel zur Verunsicherung. Der Rückgriff auf historisches Wissen diente ihm zur Untermauerung der These, dass die meisten der im positiven Recht angelegten Entscheidungen nicht zwingende Folge einer bestimmten slogischen Entwicklung seien, sondern das Ergebnis geschichtlicher Zufälligkeiten:

"Wie die Verwaltung eine zufällige, d.h. in der Staatsgeschichte gewachsene und nicht etwa schon im Wesen des Staates begründete Funktion des Staates, so ist das Verwaltungsrecht ein zufälliges, rechtsgeschichtlich gewordenes und nicht etwa schon im Wesen des Rechtes begründetes Gebiet des Rechts. «70
Merkl beschränkte sich in seinem Lehrbuch zum Allgemeinen Verwaltungsrecht nicht auf eine geschichtliche Einleitung, sondern durchsetzte sein gesamtes Werk mit zahlreichen historischen Hinweisen, um in nahezu bilderstürmerischer Art verbreitete Grundannahmen, Dogmen und Begriffe zu hinterfragen. Er wandte sich dezidiert gegen eine zu simple Einteilung der Geschichte in Phasen oder Entwicklungsstufen. Zwar griff auch Merkl auf Begriffe wie den `Verwaltungsstaat`, den 'Justizstaat`, den `Polizeistaat` oder den `Rechtsstaat` zurück. Für ihn waren dies aber Idealtypen, die nur bedingt etwas mit der historischen >Realitätı zu tun hatten.

In der geschichtlich gelebten Staatspraxis überlagerten und vermischten sich diese Typen in graduell unterschiedlichem Ausmaß. ${ }^{71}$ Merkl diente der Rückgriff auf historische Beispiele schließlich zur Illustration, dass die Ausgestaltung von Verwaltung und Verwaltungsrecht "örtlich und zeitlich schwankt «"72. Anhand eines geschichtlich orientierten Rechtsvergleichs zwischen Österreich, England, Frankreich und der Schweiz zeigte Merkl beispielsweise auf, dass sich zwischen Staatsform (Demokratie und Autokratie) und Verwaltungsorganisation (Bürokratie bzw. kollegiale Laienverwaltung) keine "natürlichen" oder "logischen" Korrelationen bilden ließen. Die Rechtsgeschichte diente der "Reinen Rechtslehre" gleichsam als Purgatorium, um die rechtliche Form oder "Schale" herauszuarbeiten und von den raumzeitlich abhängigen "Kulturinhalten « zu reinigen. ${ }^{73}$ Dieser rechtshistorisch-vergleichende Ansatz mit einem hohen Verunsicherungspotenzial stieß in der Wissenschaftsgemeinde auf wenig Gegenliebe und führte zu Irritationen. ${ }^{74}$ Das Bedürfnis nach einfachen Erzählmustern schien gerade im Verwaltungsrecht willkommen zu sein - in einer Disziplin, die wegen der Heterogenität und Volatilität ihres Forschungsgegenstandes in besonderem Maß nach Sicherheit, Stabilität und Einfachheit strebt. Im Gegensatz zu Mayer, Fleiner, Jellinek und Forsthoff fand Merkl außerhalb Österreichs kaum Beachtung und ist bis heute ein Geheimtipp für >Aficionados` des Verwaltungsrechts geblieben..$^{75}$

\section{Geschichte als Hagiografie}

Es gehört in der Rechtslehre (und nicht nur dort) zum sguten Ton`, verstorbene wissenschaftliche Größen zu 
erwähnen, auch wenn darüber hinaus kaum eine vertiefte Beschäftigung mit der eigenen disziplinären Vergangenheit stattfindet. Damit wird unterstrichen, was bewährte Lehre` ist, und der Schreibende kann sich in eine Traditionslinie illustrer Namen stellen. Das Denken in wissenschaftlichen sSchulen • beziehungsweise in personellen Kontinuitätslinien zwischen `Lehrern` und `Schülern ‘ ist gerade bei den deutschen Staatsrechtslehrern - zur Vereinigung der Deutschen Staatsrechtslehrer gehören auch die Verwaltungsrechtswissenschaftler - besonders ausgeprägt. ${ }^{76}$

Diese familien- oder zunftähnliche Formierung von Denkkollektiven führte so weit, dass sich ein juristischer Fachverlag 1999 veranlasst sah, vier >Ahnentafeln ‘ drucken zu lassen, welche in Form eigentlicher Stammbäume Vorfahren (`Lehrer`) und Nachkommen (`Schüler`) der verschiedenen Familienzweige (`Schulen`) sichtbar machen. Als Ersatz für das fehlende Familienwappen erfolgte die Hinterlegung der Stammbäume mit dem deutschen Bundesadler - ungeachtet der auch aufgeführten österreichischen und schweizerischen Personen. ${ }^{77}$ Für das Verwaltungsrecht hat Otto Mayer in besonderer Art und Weise die Rolle eines 'Stammvaters ters ${ }^{78}$ übernommen, dem in den Lehrbüchern bis heute die Referenz erwiesen wird. ${ }^{79}$ Exemplarisch erwähnt sei hier die Darstellung bei Walter Jellinek - einem 'Schüler Mayers. Die von ihm beschriebene Geschichte der Verwaltungsrechtswissenschaft dreht sich ganz um Otto Mayer und seine >Vorgänger ` und die Epigonen..$^{80}$ Neben einer eingehenden Würdigung des Werks von Mayer findet sich auch eine sehr persönlich gefärbte Hommage an den verehrten $>$ Lehrer ${ }^{81}$

Die Auseinandersetzung mit Wissenschaftlerbiografien und die Analyse ihrer familiären und wissenschaftlichen Beziehungsnetzwerke ist heute etablierter Bestandteil der Geschichte vom öffentlichen Recht. Gerade mit Blick auf die familiären Prägungen im deutschen Bildungsbürgertum, die Bedeutung der politischen, weltanschaulichen und religiösen Einstellung für das wissenschaftliche Werk und die spezifische Wissenschaftskultur im öffentlichen Recht ist dies ein aufschlussreicher Forschungsansatz. ${ }^{82}$ Dieser Zugang ist aber nicht immer frei von hagiografischen Zügen. Ein 2015 erschienener monumentaler Sammelband stellt Leben und Werk von 67 Staatsrechtslehrern
Schweiz dar. ${ }^{83}$ Das Buch ist der Vereinigung der Deutschen Staatsrechtslehrer gewidmet. Die Auswahl der 61 Autoren und 3 Autorinnen erfolgte sformal danach, »ob sie unter die Kategorie der Schülerin oder des Schülers « des jeweils Dargestellten fallen. ${ }^{84}$ Die Zahl der "Geehrten " - so werden die Porträtierten im Vorwort gleich zweimal tituliert - wurde gemäß Deklaration der Herausgeber auf höchstens 70 beschränkt. Dieses Konzept lässt erahnen, welch hagiografische Ambitionen die Herausgeber verfolgten. Die Zahl gemahnt an die 70 Ältesten Israels $(4$. Mose 11, 16) und die 70 Jünger Jesu (Lukas 10, 1-24). Und die Auswahl der Autoren in der Nachfolge ihres jeweiligen Herrn verhindert schon im Ansatz, dass sich Bilderstürmer ans Werk machen. Freilich ließ sich dieses Konzept bei den Persönlichkeiten des frühen 20. Jahrhunderts mangels noch lebender sSchüler nicht mehr umsetzen. Die entsprechenden Beiträge sind daher aus kritischer Distanz, basierend auf gut dokumentierten Quellen und den neuesten Forschungsstand berücksichtigend, verfasst. ${ }^{85}$ Demgegenüber werden die Staatsrechtslehrer, deren Wirken in die zweite Hälfte des 20. Jahrhunderts fiel, von ihren sSchülern` dargestellt. Im Vordergrund stehen Schilderungen des Charakters, prägende Ereignisse und Anekdoten. Als Berichte von Zeitzeugen haben diese Beschreibungen durchaus ihre Berechtigung. Schüler neigen gegenüber ihren verstorbenen Lehrern aber naturgemäß zu Loyalität: de mortuis nihil nisi bonum. Mit Blick auf das Verhalten gewisser Staatsrechtslehrer zwischen 1933 und 1945 erweist sich diese persönliche Befangenheit als fatal. Erwähnt sei hier nur ein Beispiel: Theodor Maunz (1901-1993) - auf ihn ist noch zurückzukommen (vgl. Abschnitt »Geschichte als Kontrastfolie «) - trat 1933 der NSDAP und der SA bei und publizierte seit 1934 zahlreiche Werke, die keinen Zweifel an seiner antisemitischen und nationalsozialistischen Gesinnung ließen. ${ }^{86}$ Der Biograf und `Schüler` widmet diesem Abschnitt in Maunz' Leben wenige Zeilen und stellt Maunz letztlich als Opfer einer Verführung dar. Zudem wird betont, dass Maunz selbst in diesem düsteren Kapitel deutscher Geschichte ein >venerabilis Dei servus blieb: ${ }^{87}$ "Doch hielt er z.B. an der stetigen Beteiligung an der Fronleichnamsprozession fest und versuchte auch sonst, manchen zu helfen. ${ }^{88}$ Dass der Biograf darauf verzichtet, kritische Sekundärliteratur über Maunz zu erwähnen, versteht sich da von selbst. 


\section{Geschichte als Kontrastfolie}

Die Befassung mit der Vergangenheit diente bei zahlreichen Autoren dazu, sich von Vergangenem emanzipatorisch abzuheben. Gerade bürgerlich-liberal geprägte Autoren benutzten den >Polizeistaat ' gerne als Kontrastfolie, vor deren Hintergrund sie ihr eigenes Programm eines rechtsstaatlichen Verwaltungsrechts entwickelten. Autoren, welche Neigungen $\mathrm{zu}$ einer heilsgeschichtlichen Betrachtungsweise haben, tendieren denn auch dazu, auf frühere Entwicklungsstufen ızurückı oder sherunter`zu blicken und so die Gegenwart und Zukunft umso strahlender erscheinen zu lassen. In seinem Lehrbuch des deutschen und preußischen Verwaltungsrechts ${ }^{89}$ formulierte dies Julius Hatschek (1872-1926) so:

»Unser deutsches Verwaltungsrecht ist vom Wesen des Rechtsstaats erfüllt. Wenn wir dies als besonderen Vorzug unseres Rechts rühmen, so stellen wir es in bewußtem Gegensatz zum früheren Polizeistaat, der dem Rechtsstaat Platz gemacht hat. Um diesen Gegensatz richtig zu würdigen, müssen wir uns vergegenwärtigen, worin das Wesen des Polizeistaats bestand. «90

Die grundlegende Abwendung von rechtsstaatlich-liberalen Konzepten nach 1933 führte in der deutschen Verwaltungsrechtswissenschaft zwangsläufig zu einem Bruch mit der Vergangenheit. Während Walter Jellinek sich dagegen vorerst noch mit einem nostalgisch verklärenden Blick in die Vergangenheit $\mathrm{zu}$ wehren versuchte, ${ }^{91}$ propagierten andere Autoren die "Überwindung" des "bürgerlich-rechtsstaatlichen Verwaltungsrechts « ${ }^{92}$. Diese »Überwindung " umfasste auch eine bewusste Abkehr von bisherigen Erzählungen und der Periodisierung der Verwaltungsgeschichte. In den Worten Theodor Maunz:

"Wir erkennen heute, daß sich in diese Dreiteilung Justizstaat, Polizeistaat und Rechtsstaat ein Übertragen von Vorstellungen späterer Zeiten auf frühere Zeiten eingeschlichen hat. [...] Angeknüpft wird nämlich an die Rechtsstellung des einzelnen und an seine mehr oder minder große Sicherung durch Rechtsnormen und Gerichte. Dadurch wird nicht die Geschichte der Verwaltung selbst in Perioden eingeteilt, sondern die
Geschichte der Stellung des einzelnen in der Verwaltung, also die Geschichte einer bürgerlich-rechtsstaatlichen Problemstellung. «93

Die an Metaphern reiche Rhetorik der liberalen Protagonisten des Verwaltungsrechts wurde gezielt aufgegriffen - allerdings mit umgekehrten Vorzeichen: Die "stehengebliebenen Trümmer «94 und die noch in die Gegenwart "ragenden Reste ${ }^{95}$ bezogen sich nun nicht mehr auf den Polizeistaat, sondern auf den Rechtsstaat. Die Abkehr von der liberal geprägten Periodisierung führte bei Claudius von Schwerin (1880-1944) dazu, sich ganz von einer übergreifenden Epochenbildung zu verabschieden und sich einer geschichtlichen Darstellung der einzelnen Verwaltungszweige zuzuwenden. ${ }^{96}$ Schwerin - der sich als Rechtshistoriker und nicht als Verwaltungsrechtswissenschaftler mit der Materie befasste - nannte als Grund für die Unmöglichkeit einer Periodisierung auch das Fehlen ausreichender Vorarbeiten im Gebiet der Verwaltungs- und Verwaltungsrechtsgeschichte und verwies auf die unübersichtlichen und schwer zugänglichen Quellenbestände. ${ }^{97}$

Demgegenüber propagierte Maunz eine "neue Dreiteilung « und gliederte die Verwaltungsgeschichte in die Epochen der »ständischen Verwaltung «, der »kameralistischen Verwaltung" und der "gesetzesstaatlichen Verwaltung ${ }^{98}{ }^{98}$ Maunz beließ es aber nicht bei dieser historischen Darstellung, sondern entwarf das Grundkonzept des "neuen« Verwaltungsrechts als bewusstes Kontrastprogramm zum »bürgerlich-rechtsstaatlichen Verwaltungsrecht «"9. Der "Gesetzmäßigkeit der Verwaltung « wurde die »Rechtmäßigkeit der Verwaltung « gegenüberstellt, der »Gewaltentrennung « der »Führergrundsatz«, dem »subjektiven öffentlichen Recht« die »Gliedstellung der Persönlichkeit« etc. ${ }^{100}$ Galt es früher, die letzten Trümmer des Polizeistaats zu identifizieren und wegzuräumen, mussten jetzt die Überreste des liberalen Rechtsstaats »überwunden « werden.

Nach 1945 knüpfte die bundesdeutsche Verwaltungsrechtswissenschaft - im Gegensatz zur DDR ${ }^{101}$ - weitgehend wieder an die Grundlagen vor 1933 und damit an die liberal-rechtsstaatliche Tradition an. Das Werk Jellineks erschien 1948 als unveränderter Nachdruck und 1950 lediglich mit einem Nachtrag. Für die Schweiz wurden mit einem 1939 erfolgten Reprint von Fleiners Institutionen bewusst die »Grundsätze des libe- 
ralen Verwaltungsrechts und damit [...] des modernen Rechtsstaates «102 hochgehalten. Die liberal geprägte Verwaltungsrechtslehre in der Nachfolge des Stammvaters Otto Mayer war damit auch nach 1945 für die Bundesrepublik und die Schweiz tonangebend. ${ }^{103}$ Von dieser Dominanz, welche eine dogmatische Erstarrung und disziplinäre Verengung möglicherweise begünstigte, sahen und sehen sich Vertreter der Verwaltungsrechtswissenschaft immer wieder herausgefordert. Forsthoff etwa bemühte sich mit seinem Konzept der Daseinsvorsorge und der Öffnung gegenüber den Verwaltungswissenschaften um eine Emanzipation. ${ }^{104}$ Seit den 1990er-Jahren tritt in Deutschland eine zunehmend dominierende Strömung in Erscheinung, welche selbstbewusst für sich in Anspruch nimmt, die »Neue Verwaltungsrechtswissenschaft « (in Großsschreibung) $\mathrm{zu}$ verkörpern. ${ }^{105}$ Die von Otto Mayer geprägte »Juristische Methode« gilt als »überkommen « und von ihr will sich die neue Strömung abheben:

"Das >Neuer an der Neuen Verwaltungsrechtswissenschaft wird erst erkennbar, wenn man sich als Kontrastfolie die wissenschaftliche Arbeitsweise nach der sog. Juristischen Methode vergegenwärtigt. «106

Die "Neue Verwaltungsrechtswissenschaft« erhebt unter anderem den Anspruch, in erhöhtem Maße wissenschaftlich, interdisziplinär und steuerungsorientiert zu sein. Die stark dogmatisch und auf den Einzelfall fokussierte Ausrichtung auf die Interpretation des geltenden Rechts (juristische Methode) soll einer sozialwissenschaftlich fundierten Sichtweise über das zukünftig richtige Recht weichen. ${ }^{107}$ Mit diesem Perspektivenwechsel soll ıneuen، Entwicklungen wie der Privatisierung, alternativen Steuerungsformen und der Internationalisierung Rechnung getragen werden.

Diese Forderungen sind in gewisser Hinsicht durchaus berechtigt. ${ }^{108}$ Das in vielen dieser Publikationen mitschwingende Narrativ, wonach die juristische Methode "überkommen « sei und die tradierte Verwaltungsrechtswissenschaft in einer "Krise« stecke, neigt aber zu einer vereinfachenden Darstellung. Als Kontrastfolie ist die Legende von der dogmatisch verkrusteten und sozialwissenschaftlich ignoranten Disziplin jedoch bestens geeignet, um den eigenen wissenschaftlichen Anspruch und den innovativen Gestaltungswillen zu unterstrei- chen. ${ }^{109}$ Letztlich tat Otto Mayer dasselbe, als er sich von der »überkommenen« Kameralistik des Polizeistaats abhob und das Verwaltungsrecht als "ebenbürtige« Wissenschaftsdisziplin nach dem Vorbild der etablierten Zivilrechtswissenschaft begründen wollte. ${ }^{110}$ Hier treffen sich alt und neu: Otto Mayer wie auch die Vertreter der "Neuen Verwaltungsrechtswissenschaft « unterstreichen durch die kontrastierende Stilisierung der disziplinären Vergangenheit den Innovationsgehalt der eigenen Tätigkeit und machen ihre Geschichtsschreibung zum Bestandteil einer erfolgreichen Wissenschaftsstrategie. ${ }^{111}$

\section{Geschichtsvergessenheit}

Lässt man die - meist von Rechtshistorikern verfasste Spezialliteratur beiseite, so fällt auf, dass zahlreiche der heute verbreiteten Lehrbücher zum Verwaltungsrecht ohne historische Einleitungen auskommen und auch sonst den geschichtlichen Hintergrund ausblenden. Dies gilt für Deutschland ${ }^{112}$ und in Extremform für die Schweiz ${ }^{113}$. Etwas anders präsentiert sich die Lage in Österreich. ${ }^{114}$ Für diesen zurückhaltenden Umgang mit der Geschichte gibt es verschiedene Erklärungsansätze. ${ }^{115}$

Mit Blick auf die Bundesrepublik scheint ein wesentlicher Grund für diese Entwicklung, dass die verfassungsrechtliche Perspektive nach 1949 zu einer Neuausrichtung der Disziplin führte. Das Verwaltungsrecht musste seine Legitimität nicht mehr aus historischer Kontinuität schöpfen, sondern aus der Rückbindung an die Verfassung. ${ }^{116}$ Das Grundgesetz, dieses »juristische Regelwerk von zeitloser Gültigkeit «117, wurde nun zum Garanten von Stabilität und Kontinuität. Daneben mag ein anderer, biografisch-psychologischer Grund der Geschichtsblindheit Vorschub geleistet haben. Viele der deutschen Staatsrechtslehrer hatten zwischen 1933 und 1945 in unterschiedlichem Ausmaß mit nationalsozialistischem Gedankengut sympathisiert oder dieses gar - wie im Falle von Maunz - offen propagiert. Dieser Umstand führte in der jungen Bundesrepublik - auch von Seiten weniger kompromittierter Kollegen ${ }^{118}$ - zu einer "kollegialen Solidarität des Verschweigens «119 in Bezug auf die Vergangenheit und ist in Ausläufern bis heute präsent. ${ }^{120}$ Ein "Schleier der Harmonie über Unauflösbares « ${ }^{121}$ breitete sich aus, und der Blick wurde im Interesse des Wiederaufbaus nach vorne gerichtet: 
"Mit diesen Erkenntnissen, die wir keinen Augenblick vergessen dürfen, ziehen wir einen Schlussstrich unter die Vergangenheit. So wichtig das Wissen um das Negative ist, darf es uns nicht vor positiver Aufbauarbeit abhalten. «122

Die Enthistorisierung kann aber nicht alleine auf die besonderen Umstände in Deutschland nach 1945 zurückgeführt werden. Der Erklärungsansatz mit der nationalsozialistisch `belasteten Vergangenheit taugt etwa für die Schweiz nicht; gerade die schweizerischen Lehrbücher kommen aber ohne jeden Bezug zur Vergangenheit aus. Eine Erklärung ist, dass das Verwaltungsrecht - im Gegensatz zum Verfassungsrecht - gerne als apolitisch, technisch und damit gegenüber historischen Veränderungen weitgehend immun betrachtet wird. Otto Mayer legte mit seinem berühmten Diktum (»Verfassungsrecht vergeht, Verwaltungsrecht besteht «123) den Grundstein $\mathrm{zu}$ dieser Betrachtungsweise, die spätere Autoren bereitwillig aufgriffen. ${ }^{124}$ Möglicherweise ist das Bedürfnis der Rechtswissenschaft nach `Entpolitisierung des Verwaltungsrechts in einem Land wie der Schweiz gerade deshalb so ausgeprägt, weil die Sphären zwischen >Politikı und `Verwaltung ‘ traditionell weniger klar trennen lassen als anderswo. ${ }^{125}$

Neben dem scheinbar apolitischen Charakter des Verwaltungsrechts ist es schließlich der Systemgedanke, der einer historischen Betrachtungsweise nur beschränkt zugänglich ist. Mittels der Bildung von Systemen versucht die Verwaltungsrechtswissenschaft in einem "Chaos von einzelnen Gesetzesparagraphen «126 Ordnung, Orientierung und festen Halt zu bieten. Der Systemgedanke nimmt dabei eine zentrale Stellung ein. Dies gilt nicht nur für die Verwaltungsrechtswissenschaft im Sinne der traditionellen Dogmatik, ${ }^{127}$ sondern ebenso für die "Neue Verwaltungsrechtswissenschaft « ${ }^{128}$. Systeme sollen dazu dienen, »die einzelnen Erscheinungen des Allgemeinen Verwaltungsrechts als Teile einer überlegten Konstruktion zu begreifen « ${ }^{129}$. Systembildung ist somit auch Arbeit an einer kohärenten und widerspruchsfreien 'Rechtsordnung ‘. ${ }^{130}$ Betrachtet man das Verwaltungsrecht aus einer historischen Perspektive, so gerät dieser Systemgedanke ins Wanken: Die Verwaltung und auch das Verwaltungsrecht wurden nicht auf dem Reißbrett als in sich stim- dern sind das Ergebnis politischer, gesellschaftlicher und wirtschaftlicher Entwicklungen, Streitigkeiten und Aushandlungsprozesse.

Die politischen Diskurse, vor deren Hintergrund Verwaltungsrecht serzeugt ‘ wird, verlaufen je nach Zeit, Ort und Sachgebiet anders. Und auch die unterschiedlichen Ebenen des Gemeinwesens, welche aufgrund ihrer Zuständigkeiten an diesem Erzeugungsvorgang beteiligt sind (supranationale Ebene, Bund, Kantone bzw. Länder, Gemeinden), führen zwangsläufig zu einem rechtlichen Pluralismus. ${ }^{131}$ Wer den systematischen Charakter des Verwaltungsrechts betont, wird bewusst oder unbewusst dazu neigen, die historisch gewachsene Realität mit all ihren Widersprüchlichkeiten auszublenden.

Dass der Systemgedanke einer enthistorisierenden Betrachtungsweise förderlich ist, zeigt gerade der Vergleich mit dem angelsächsischen Rechtskreis. Im Common Law erfolgt der traditionelle Zugang zu einer Rechtsfrage über die Gerichtspraxis. Gedacht wird in Rechtsprechungslinien, deren Wurzeln teilweise bis ins 17. oder 18. Jahrhundert zurückreichen. Jedes Urteil bezieht sich sodann auf einen bestimmten Streitfall und muss zuerst kontextualisiert werden, um es auf neue Fallkonstellationen anwendbar zu machen. Argumentiert wird - anders als im kontinentaleuropäischen Rechtsraum - nicht unter deduktiver Verwendung von Begriffen, Systemen und Prinzipien, sondern mittels induktivem "reasoning from case to case «. ${ }^{132}$ Der Mangel an begrifflicher Schärfe und systematischer Durchdringung mag aus kontinentaleuropäischer Sicht als Rückständigkeit erscheinen. ${ }^{133}$ Gehört das Erzählen von »Mikrogeschichten « aber zum Kerngeschäft der Juristinnen und Juristen, so führt dies auch zu einer intensiveren wissenschaftlichen Auseinandersetzung im historischen Kontext und damit zur Befassung mit der »Makrogeschichte «. ${ }^{134}$ Mit ihrer evolutionären Denkweise unterscheidet sich die angelsächsische Rechtskultur damit grundlegend von dem zur Erstarrung neigenden Systemdenken kontinentaleuropäischer Prägung. ${ }^{135}$

Ein letzter Grund für das Verschwinden der Geschichte aus den Lehrbüchern des Verwaltungsrechts mag sodann eine besonders für Deutschland zu beobachtende Ausdifferenzierung der juristischen Literaturgattungen sein. Auf der einen Seite nimmt die wissenschaftliche Auseinandersetzung mit der Geschichte von Verwaltung, Verwaltungsrecht und Verwaltungsrechtswissenschaft 
zu und findet Ausdruck in umfangreichen Publikationen mit ausgebautem wissenschaftlichem Apparat.

Es sind Werke von Wissenschaftlern für Wissenschaftler. Diese enthalten meist auch profunde Beiträge zur Geschichte von Verwaltungsrecht und Verwaltungsrechtswissenschaft, in der Regel verfasst von Rechtshistorikern. ${ }^{136}$ Daneben sterben die klassischen `Lehrbücher`, welche die Grundlage für den universitären Unterricht bilden und zugleich wissenschaftlichen Anspruch erheben, aus. Entweder mutieren sie zu umfangreichen und mehrbändigen Werken und kommen damit für den Unterricht kaum mehr infrage. ${ }^{137}$ Oder sie werden verdrängt durch `Lernbücher`, welche keinen wissenschaftlichen Anspruch mehr erheben und deren Abfassung auch kein Prestige in der akademischen Welt verspricht. Diese Literaturgattung dient in erster Linie der zielgerichteten Vorbereitung auf die Staatsexamen. Im Vordergrund steht daher die Vermittlung von Wissen, das für die praktische Berufsausübung in Anwaltschaft und Justiz relevant erscheint. Und dieses Wissen bezieht sich ausschließlich auf das geltende Recht. Die Befassung mit Rechtsgeschichte - sei dies als eigenständiges Fach oder als Hinführung zu einem Kurs über aktuelle Rechtsfragen - belastet die ohnehin reich befrachtete Lernagenda nur unnötig: Man braucht so etwas nicht. ${ }^{138}$

\section{Zum Schluss: Warum die Verwaltungsrechtswissenschaft Geschichte mitschreiben sollte}

Nach dieser Analyse in Gestalt eines Rückblicks (einer Art Meta-Geschichte) soll hier versucht werden, eine eigene Antwort auf die Frage zu finden, warum und wie sich die Verwaltungsrechtswissenschaft mit der Geschichte von Verwaltung, Verwaltungsrecht und mit der Vergangenheit der eigenen Disziplin befassen soll.

\section{Erster Grund: historisches Wissen als Erklärungs- und Verständnishilfe}

Die Verwaltungsrechtswissenschaft ist innerhalb der Jurisprudenz eine vergleichsweise junge Disziplin. Dennoch scheinen ihre eigenen Wurzeln und die Geschichte und der Blick in besonderem Maß dem geltenden Recht zugewandt. Der Verzicht auf eine historische Perspektive scheint gut verkraftbar, sieht doch ein Großteil der Verwaltungsrechtswissenschaft ihre Hauptaufgabe darin, praktikable Lösungsansätze für Gegenwartsprobleme bereitzustellen. Es mag paradox erscheinen - doch möglicherweise ist es gerade diese, in der heutigen Verwaltungsrechtswissenschaft vorherrschende enthistorisierte Sichtweise, die das Beharrungsvermögen gewisser Denkstrukturen und eine unbewusste Traditionsgebundenheit fördert. ${ }^{139}$ Das Denken in abstrakten Begriffen und Systemen erscheint mitunter als eine von Raum und Zeit abgehobene, technokratische Operation. Dabei wird vergessen, dass die Verwaltung, das Verwaltungsrecht und die Verwaltungsrechtswissenschaft in einem bestimmten zeitlichen Kontext entstanden sind und sich ständig weiterentwickeln.

Gerade in Kombination mit der Rechtsvergleichung erlaubt die Beschäftigung mit der Geschichte, die Gegenwart besser in einen raumzeitlichen Kontext einzuordnen und zu verstehen. Verstehen meint nicht, dass sich konkrete ‘Lehren vom Baum der Erkenntnis pflücken ließen. ${ }^{140}$ Direkter `Nutzen ‘ für das gegenwärtige Recht und seine Anwendung lässt sich - einmal abgesehen von der historischen Auslegung - aus der Beschäftigung mit der Geschichte selten ziehen. ${ }^{141}$ Wenn es aber ein zentrales Anliegen der Verwaltungsrechtswissenschaft ist, in einem hochgradig heterogenen und volatilen Umfeld Orientierung zu bieten, dann kann die Geschichte einen wertvollen Beitrag hierzu liefern. Weniger im Sinne einer >Rückführung ‘ auf vorbestehende und unverbrüchliche "juristische Grundlinien « ${ }^{142}$. Eher geht es darum, die Dogmen, Begriffe und Institutionen des heutigen Verwaltungsrechts als Antworten auf bestimmte Herausforderungen in der Vergangenheit $\mathrm{zu}$ verstehen. Diese Kontextualisierung vermag meist mehr zu erklären, als der Versuch, die heterogenen Erscheinungen des Rechtsalltags in ein System zu zwängen und sich um eine vermeintlich widerspruchsfreie Rechtsordnung zu bemühen.

Die Historisierung des Verwaltungsrechts kann somit ausgleichen, was eine Systematisierung nicht zu leisten vermag. Sie hilft, mit Widersprüchen und Inkonsequenzen umzugehen - und gerade hiervon ist das Verwaltungsrecht überreich. Das Verwaltungsrecht und auch die Verwaltungsrechtswissenschaft wurden wiederholt 
mit einem Gebäude verglichen. ${ }^{143}$ Diese Metapher kann für das Verstehen der Materie hilfreich sein. Allerdings nur dann, wenn man sich ein Schloss vorstellt, an dem Generationen gebaut haben und das eine verwirrende Vielzahl von Zimmern, Sälen, Treppenhäusern, Kellergewölben, Dachkammern und zahlreichen Um- und Anbauten aufweist. ${ }^{144}$ Wer dieses Gebilde vorsichtig und neugierig erkundet und einen Sinn für Architekturgeschichte entwickelt, wird sich darin schneller orientieren als derjenige, der krampfhaft darum bemüht ist, den logischen Bauplan eines modernen Verwaltungsgebäudes zu erkennen. Die Geschichte kann so als Erklärungs- und Verständnishilfe dienen und verhindert eine vorschnelle systematische Vereinnahmung.

\section{Zweiter Grund: Geschichte zur Förderung von Respekt und Verblüffungsresistenz}

So wie die Befassung mit der Vergangenheit Verständnis für die Komplexität der Materie Verwaltung oder Verwaltungsrecht wecken kann, so kann die Auseinandersetzung mit der Geschichte der eigenen Disziplin Verständnis wecken, aber auch Respekt und Verblüffungsresistenz. Wer sein >wissenschaftsgeschichtliches Gedächtnis` pflegt, dem wird eher bewusst, dass wissenschaftliche Erkenntnis keine individuelle Eigenleistung darstellt, sondern immer auf dem aufbaut, was in der Vergangenheit geleistet wurde. Forschung beginnt bildlich gesprochen - kaum je auf der 'grünen Wiese`, sondern ist meist das Erweitern oder Renovieren eines bestehenden Gebäudes. Zudem sahen sich auch frühere Generationen mit vergleichbaren Fragestellungen konfrontiert und bemühten sich ernsthaft um überzeugende Lösungsansätze. Die damit geförderte Grundhaltung mag man als Respekt oder - vielleicht altmodisch - als Demut bezeichnen. Rechtsgeschichte kann so eine ähnliche Funktion erfüllen wie Rechtsvergleichung. Das Eintauchen in die Andersartigkeit einer räumlich oder zeitlich von uns entfernten Rechtskultur fördert den Respekt vor diesem Anderssein und schützt vor Überheblichkeit gegenüber dem vermeintlich `Rückständigen`.

Der latente Druck, dass Forschung - auch in den Geistes- und Sozialwissenschaften - >innovativ < zu sein hat, macht ein 'wissenschaftsgeschichtliches Gedächtnis» besonders wichtig. Wie im Abschnitt "Geschichte als Kontrastfolie» gezeigt wurde, neigt jede Generati- on von Wissenschaftlern dazu, die eigene Erkenntnis mit dem Etikett `neu ‘ zu versehen und >Reformen` von ,Überkommenem` zu fordern. Sei es, weil Verwaltung und Verwaltungsrecht vor gefühlten oder tatsächlichen Entwicklungen und Herausforderungen (Gesetzesflut, Privatisierung, Internationalisierung, Entstaatlichung, Atomisierung der Verwaltung) stehen, weil diese Problemlagen von der Wissenschaft erstmals erkannt werden oder weil nun endlich die disziplinär geschärfte, trans- oder interdisziplinäre Methode gefunden wurde, mit deren Hilfe die Probleme bewältigbar werden.

Wer den Innovationsgehalt wissenschaftlicher Forschung beurteilen will, muss daher nicht nur die neuesten Publikationen seiner eigenen Disziplin kennen, sondern sollte sich auch mit den wissenschaftlichen Theorien, Methoden und Ansätzen befassen, die vor 50 oder 100 Jahren sen vogue Europäisierung und Internationalisierung des Verwaltungsrechts einen Beitrag zum >Global Administrative Law leisten will, sollte sich bewusst sein, dass Konzepte, Begriffe und Systeme in jeder Rechtskultur eine andere historische Verwurzelung haben. Zugleich macht gerade eine historische Betrachtung deutlich, wie schwierig die Attribute >fremd und seigen ‘ im Recht sind. ${ }^{145}$ Die so geförderte Verblüffungsresistenz darf freilich nicht in eine konservativ-abwehrende Grundskepsis umschlagen, wonach es grundsätzlich nichts Neues unter der Sonne gibt oder mit deren Hilfe jede transnationale Debatte über Verwaltungsrecht im Keime erstickt wird.

\section{Voraussetzung: Befassung mit der allgemeinen Geschichte}

Die Auseinandersetzung mit der Vergangenheit von Verwaltung, Verwaltungsrecht und Verwaltungsrechtswissenschaft bedingt ein Mindestmaß an Befassung mit der allgemeinen Geschichte. Wer sich ausschließlich mit spezifisch juristischen Textkategorien früherer Zeiten - Gesetzestexten, Urteilen oder juristischen Lehrbüchern - beschäftigt, setzt sich der Gefahr aus, in diese Quellen sein heutiges Rechts- und Staatsverständnis hineinzulesen und nur das zu erkennen, was uns bereits vertraut ist. ${ }^{146}$ Gerade im Verwaltungsrecht müssen die Quellen in ihrem politischen und ideengeschichtlichen Kontext verstanden und dargestellt werden, damit sie für das heutige Denken anschlussfähig 
werden. ${ }^{147}$ Die Befassung mit der allgemeinen Geschichte fördert zudem eine Zurückhaltung gegenüber einer allzu vereinfachenden Epochenbildung. Begriffe wie 'paternalistischer Staat‘, ’Nachtwächterstaat‘, ১liberaler Rechtsstaatı, ১Polizeistaat‘, ১Wohlfahrts- oder Sozialstaat` oder `Gewährleistungsstaat` sollen deswegen aber nicht aus dem Vokabular verbannt werden. Sie sollten viel eher im Merklschen Sinn als Prototypen verstanden werden und nicht als Beschreibungen eines empirisch nachgewiesenen historischen Befunds. ${ }^{148}$

Die Aufforderung, sich mit allgemeiner Geschichte zu befassen, soll nicht dahingehend verstanden werden, es sei nun Aufgabe der Verwaltungsrechtswissenschaftler, einen aktiven Beitrag zu deren Schreibung zu leisten. Die allgemeine Geschichtsschreibung ist in erster Linie eine Aufgabe der Historikerinnen und Historiker. Wünschenswert wäre aber, dass die Rechtswissenschaft ihre Bedürfnisse nach historischer Forschung - etwa im Bereich der Verwaltungs- oder politischen Ideengeschichte - artikuliert und die Geschichtswissenschaft ein offenes Ohr für solche Anregungen hat. ${ }^{149}$

\section{Erste Konsequenz: die unvermeidlichen Narrative}

Die hier geschilderten Beispiele aus der verwaltungsrechtlichen Literatur haben es deutlich gezeigt: Das Erzählen von Geschichten ist attraktiv. ${ }^{150}$ Wer die Vergangenheit in eine stimmige Erzählung verpacken kann, wird - gerade auch bei Nicht-Historikern - eher Anklang finden. Solche Erzählstränge können in einer klaren Epocheneinteilung (z. B. bei Jellinek), in der Darstellung von auf- oder absteigenden »Entwicklungsstufen « (etwa bei Otto Mayer) oder der Fokussierung auf eine zentrale Rezeptionsquelle des modernen Verwaltungsrechts (Verwaltungsrecht als »Oeuvre de la France «151) bestehen. Diese Erzählungen sind dankbar, weil sie helfen, Komplexität zu reduzieren und Orientierung zu vermitteln. Sie erfüllen damit eine ähnliche Funktion wie die Systematisierung. Genau wie dieser ist diesen sgroßen Erzählungen a aber ein hohes Maß an Konstruktion und Abstraktion eigen. Ihre größte Stärke - die Vereinfachung - ist zugleich ihre größte Schwäche.

Dennoch lässt es sich kaum vermeiden, dass Geschichtsschreibung bis zu einem gewissen Grad auch das Erzählen von Geschichten ist. Ohne eine minimale
Erzählstruktur, ohne Anfang und Ende, ohne das Herausarbeiten markanter Geschehnisse, ohne das Aufzeigen von Wechselwirkungen und Einflüssen und ohne Übersetzungsarbeit für uns heutige Leserinnen und Leser wird historische Forschung zum konzeptlosen und unübersichtlichen Anhäufen von Quellenmaterial. ${ }^{152}$ Dieses Erzählen ist nicht schon deswegen unwissenschaftlich, weil es verschiedene Narrative gibt und keine Erzählung für sich den Anspruch erheben kann, zu beschreiben, wie es `wirklich war`. Konträre Erzählungen und Gegenerzählungen sind für die Wissenschaftskultur sogar von zentraler Bedeutung. ${ }^{153}$

In dieser Hinsicht gleicht die Geschichtsschreibung der juristischen Auslegungsmethode: Ausgangspunkt ist in beiden Fällen eine Quelle, in der Regel ein Text (z. B. Gesetzestexte, behördliche oder gerichtliche Entscheidungen) - seltener eine bildliche Darstellung (etwa ein Organigramm der Verwaltung) oder ein Objekt (beispielsweise ein Verwaltungsgebäude). ${ }^{154}$ Natürlich lässt sich auch darüber streiten, ob diese Quellen secht ‘ sind, etwa bei mittelalterlichen Urkunden. Gerade in der neueren Verwaltungs- und Verwaltungsrechtsgeschichte dürfte dieser Punkt aber kaum kontrovers sein. Die Quelle als solche steht meist fest und über sie kann sich die Wissenschaft nicht hinwegsetzen. Insofern gibt es ein »Vetorecht der Quellen « ${ }^{155}$.

Wie diese Quellen gedeutet und zu anderen Quellen in Bezug gesetzt (kontextualisiert) werden, ist eine Frage der Interpretation. Juristen wie Historiker interpretieren Quellen und schreiben Geschichten. Sie serfinden، diese Geschichten aber nicht, sondern konstruieren sie anhand von nachvollziehbaren Methoden und machen sie mit dem Verweis auf Quellen für Dritte überprüfbar. ${ }^{156}$ Die Auswahl der Quellen und Methodenvielfalt in der Geschichts- wie Rechtswissenschaft ${ }^{157}$ bringt es mit sich, dass es >dier Geschichte nicht gibt, sondern nur unterschiedliche Erzählungen.

\section{Zweite Konsequenz: Geschichtsschreibung als Dogmen-, Begriffs- und Institutionengeschichte}

Der Gefahr, in einen allzu vereinfachenden Erzählmodus zu geraten, kann in der Verwaltungsrechtsgeschichte am ehesten dadurch begegnet werden, indem rechtshistorische Forschung stärker fokussiert wird. An 
die Stelle des Gestus der 'großen Erzählung، sollte das Erzählen möglichst konkreter und quellenbasierter Geschichten treten. Im Vordergrund stehen für das Verwaltungsrecht eine Dogmen- und Begriffsgeschichte. Denn die Verwaltungsrechtslehre ist in besonderem Ausmaß geprägt von scheinbar unumstößlichen Lehrsätzen oder Axiomen. Etwa dem Gesetzmäßigkeits- ${ }^{158}$ oder Verhältnismäßigkeitsprinzip ${ }^{159}$, dem Hierarchieprinzip ${ }^{160}$ oder der Vorstellung von einem verwaltungsrechtlichen Innen- und Außenverhältnis. Sie dominieren das rechtswissenschaftliche Denken in all seinen Facetten.

Ähnliches gilt für Begriffe, wie etwa den Verwaltungsakt (bzw. die Verfügung [Schweiz] ${ }^{161}$ oder den Bescheid [Österreich]), das Ermessen ${ }^{162}$, die Anstalt, das subjektive Recht ${ }^{163}$, das Monopol oder die Polizeigüter ${ }^{164}$. Gerade weil diese Dogmen und Begriffe meist nicht in Rechtsnormen kodifiziert sind und im Alltag kaum je hinterfragt werden, besteht eine Tendenz zur Erstarrung. Eine Dogmen- und Begriffsgeschichte kann einen Beitrag dazu leisten, diese dogmatischen Verkrustungen aufzubrechen und unbewusste Traditionsbindungen transparent zu machen. ${ }^{165} \mathrm{Im}$ Vordergrund steht dann weniger eine allgemeine Epochenbildung oder eine das gesamte Verwaltungsrecht erfassende Darstellung von Entwicklungslinien. Vielmehr sollen punktuell »längsschnittartige Entwicklungsabläufe« aufgezeigt werden. ${ }^{166}$ Das Ziel muss dabei nicht - wie bei Merkl - die vollständige Dekonstruktion sein. ${ }^{167}$ Die Verwaltungsrechtswissenschaft ist wegen des raschen Wandels ihres Gegenstands auf eine kontinuierlich-evolutionäre Weiterentwicklung im Sinne Fleiners angewiesen. Ein dogmen- und begriffsgeschichtlicher Zugang kann aber helfen, ausgetretene Pfade zu erkennen und eine behutsame und reflektierte Richtungskorrektur einzuschlagen.

Das Verwaltungsrecht ist schließlich - stärker als etwa das Zivilrecht - geprägt von Institutionen, seien es Verwaltungsbehörden oder Gerichte. In ganz besonderem Maß trifft dies für den Conseil d'Etat und seinen Einfluss auf das französische Verwaltungsrecht $\mathrm{zu}^{168}$ Aber auch im deutschsprachigen Raum haben Institutionen wie das Preußische Oberverwaltungsgericht ${ }^{169}$, der österreichische Verwaltungsgerichtshof ${ }^{170}$ oder das Schweizer Bundesgericht ${ }^{171}$ das Verwaltungsrecht und seine Weiterentwicklung geprägt. Solche Organisationen näher zu untersuchen - zum Beispiel ihre inneren Strukturen und Verfahren, ihre personelle Zusammensetzung oder ihre Praktiken in Verwaltung und Rechtsprechung - kann helfen, die Funktionsweise von Verwaltung und Justiz und damit auch die Wirkungsweise des Verwaltungsrechts in einem breiteren Kontext zu verstehen. Institutionengeschichte kann aufzeigen, dass Verwaltung und Justiz mehr sind als bloße sSubsumtionsautomaten oder 'Durchlauferhitzer` der Politik, sondern einen wesentlichen Beitrag zur Politik- oder Rechtsgestaltung in einem ganzheitlichen Sinn leisten. ${ }^{172}$

Eine Fokussierung auf dogmen-, begriffs- und institutionengeschichtliche Aspekte hat den weiteren Vorteil, dass sie für Juristinnen und Juristen mit den ihnen zur Verfügung stehenden Mitteln und Methoden bewältigbar ist. Im Vordergrund steht die Arbeit mit 'Rechtsquellen` (Verfassungstexte, Gesetzestexte, Verordnungstexte, Reglemente etc.), Entscheiden von Verwaltungsbehörden und Gerichten, sogenannte 'Materialien der Gesetzgebung`, sowie die Befassung mit juristischer Fachliteratur. Das Auffinden und der Umgang mit diesen Quellen gehört zum Kerngeschäft der Rechtswissenschaften. Dieser disziplinär geschärfte Fokus schließt nicht aus, dass zusätzliche Gesichtspunkte, wie die Kulturgeschichte (Technik der Datenverarbeitung, Kommunikation, Repräsentation, Architektur) oder biografische Hintergründe ausgeleuchtet werden. Es wäre sogar ausgesprochen wünschenswert, wenn gerade Allgemeinhistorikerinnen und -historiker diese Forschungsfelder komplementär abdeckten. ${ }^{173}$ Denn ein monodisziplinär-juristischer Zugang zur Materie birgt auch die Gefahr, sich zu sehr durch ein bereits eingeimpftes dogmatisch-begriffliches Vorverständnis kanalisieren zu lassen. ${ }^{174}$

\section{Schlussbemerkung: Juristen als "Amateurhistoriker«?}

Wer sich als Vertreter der Verwaltungsrechtswissenschaft mit der Vergangenheit von Verwaltung, Verwaltungsrecht oder Verwaltungsrechtswissenschaft beschäftigt, riskiert den Ruf, sich als "Amateurhistoriker « ${ }^{175}$ profilieren zu wollen. Man kann dies als Kompliment verstehen, drückt der Begriff 'Amateur rein sprachlich doch die Liebe zum Fach aus. Und diese scheint für wissenschaftlich tätige Personen nicht nur wünschenswert, sondern unverzichtbar. Wenn man im Amateur aber das Gegenstück zum 'Profiı sieht, so ist die Zuordnung in die eine oder andere Schublade zu- 
mindest für die Wissenschaftsgeschichte fragwürdig. Wer Wissenschaftsgeschichte betreibt, muss nicht nur im Umgang mit der Vergangenheit geschult sein. Zugleich muss die Person vertiefte Kenntnisse dieser Disziplin mitbringen und die jeweiligen disziplinären Fragestellungen und Methoden kennen.

Während Historikerinnen und Historiker besser verstehen, wie mit zeitlicher Distanz umzugehen ist, stoßen sie wegen fachlicher Distanz zu ihrem Forschungsgegenstand an Grenzen. Medizingeschichte ohne medizinische Grundkenntnisse ist ebenso samateurhaft ‘ wie Kirchengeschichte ohne theologischen Hintergrund. Juristinnen und Juristen sollten daher nicht davor zurückschrecken, sich mit ihrer eigenen Vergangenheit zu beschäftigen und damit einen Beitrag zur Geschichtsschreibung zu leisten. In diesem Sinn endet dieser Beitrag mit dem Ratschlag aus einer Rektoratsrede des Theologen und Kirchenhistorikers Adolf Harnack (1851-1930):
»Ich bin am Schluss, aber ein Doppeltes möchte ich Ihnen, meine Commilitonen, noch ans Herz legen: erstlich, was Sie auch studieren mögen, vernachlässigen Sie die Geschichte nicht, die große Geschichte und die ihrer Wissenschaft. Glauben Sie nicht, dass Sie Erkenntnisse einsammeln können, ohne sich mit den Persönlichkeiten innerlich zu berühren, denen man sie verdankt, und ohne den Weg zu kennen, auf dem sie gefunden worden sind. Keine höhere wissenschaftliche Erkenntnis ist eine bloße Thatsache; eine jede ist einmal erlebt worden, und an dem Erlebniss haftet der Bildungswerth. Wer sich damit begnügt, nur die Resultate sich anzueignen, gleicht dem Gärtner, der seinen Garten mit abgeschnittenen Blumen bepflanzt. «176 
A C K E R M A N N , Christian: Die Bedeutung der Rechtsprechung des Preußischen Oberverwaltungsgerichts zum Kommunalrecht für unsere heutige Dogmatik, Baden-Baden 2012. A D A M O V I C H, Ludwig K. / et al.: Österreichisches Staatsrecht, Bd. 4: Allgemeine Lehren des Verwaltungsrechts, Wien 2009. A E R S C H M A N N , Stephan: Der ideale Richter: Schweizer Bundesrichter in der medialen Öffentlichkeit (18752010), Zürich 2014. A N T O N I O L L I, Walter / K O J A, Friedrich: Allgemeines Verwaltungsrecht, Wien ${ }^{31996 . ~ B ~ A ~ B ~ E ~ R ~ O ~ W ~ S ~ K ~ I, ~ J o ̈ r g: ~ D e r ~}$ Sinn der Geschichte: Geschichtstheorien von Hegel bis Foucault, München 2005. B I A G G I N I, Giovanni: „Fritz Fleiner (1867-1937)«, in: Peter Häberle, Michael Kilian, Heinrich Amadeus Wolff (Hg.), Staatsrechtslehrer des 20. Jahrhunderts, Berlin 2015, S. 110-126. B O G D A N DY, Armin von, "Verwaltungsrecht im europäischen Rechtsraum - Perspektiven einer Disziplin«, in: Ders., Sabino Cassese, Peter M. Huber (Hg.), Handbuch Ius Publicum Europaeum, Bd. IV, Heidelberg 2011, S. 3-36. B O L D T, Hans / S T O L L E IS, Michael: "Geschichte der Polizei in Deutschland«, in: Hans Lisken, Erhard Denninger (Hg.), Handbuch des Polizeirechts, München ${ }^{4} 2007$, S. 1-41. B R O H M , Winfried: "Die Dogmatik des Verwaltungsrechts vor den Gegenwartsaufgaben der Verwaltung", in: VVDStRL 30 (1971), S. 245-364. B U M K E, Christian: „Die Entwicklung der verwaltungsrechtswissenschaftlichen Methodik in der Bundesrepublik Deutschland«, in: Eberhard SchmidtAßmann, Wolfgang Hoffmann-Riem (Hg.), Methoden der Verwaltungsrechtswissenschaft, Baden-Baden 2004, S. 73-130. C A N C I K, Pascale: "Verwaltungsrechtsgeschichte», in: Rechtsgeschichte 19 (2011), S. 30-34. - "Diskussionsvotum an der Jahrestagung der Vereinigung der Deutschen Staatsrechtslehrer 2015«, in: VVDStRL 75 (2016), S. 267 f. C A R O N I, Pio: Die Einsamkeit des Rechtshistorikers, Basel 2005. C O UTAZ, Gilbert / et al.: "Was soll und kann Verwaltungsgeschichte?», in: traverse 3 (2011), S. 160-170. D A N W I T Z, Thomas von: Europäisches Verwaltungsrecht, Berlin 2008. D E N N E W I T Z, Bodo: Die Systeme des Verwaltungsrechts, Hamburg 1948. D O M M A N N, Monika / G U GERLI, David: "Geschichtswissenschaft in Begutachtung: Acht Kommentare zur historischen Methode der Gegenwart", in: traverse 2 (2011), S. 154-164. D R E I E R, Horst: »Merkls Verwaltungsrechtslehre und die deutsche Dogmatik des Verwaltungsrechts", in: Robert Walter (Hg.), Adolf J. Merkl: Werk und Wirksamkeit, Wien 1990, S. 55-88. - Hierarchische Verwaltung im modernen Staat: Genese, aktuelle Bedeutung und funktionelle Grenzen eines Bauprinzips der Exekutive, Tübingen 1991. E H L E R S, Dirk: »Otto Mayer (1846-1924)«, in: Peter Häberle, Michael Kilian, Heinrich Amadeus Wolff (Hg.), Staatsrechtslehrer des 20. Jahrhunderts, Berlin 2015, S. 46-56. E N G E L, Christoph: "Herrschaftsausübung bei offener Wirklichkeitsdefinition«, in: Ders., Wolfgang Schön (Hg.), Das Proprium der Rechtswissenschaft, Tübingen 2007, S. 205-240. E R R A S S , Christoph: »Zur Geschichte des Bundesgerichts", in: Marcel Alexander Niggli, Peter Uebersax, Hans Wiprächtiger (Hg.), Basler Kommentar zum Bundesgerichtsgesetz, Basel 22011, S. 1-77. E S S E N, Georg / J A N SE N, Nils (Hg.): Dogmatisierungsprozesse in Recht und Religion, Tübingen 2011. F E R G U S O N, Niall: The Great Degeneration: How Institutions Decay and Economies Die, London 2012. F L E I N E R, Fritz, Schweizerisches Bundesstaatsrecht, Tübingen 1923. • "Beamtenstaat und Volksstaat» (aus der Festgabe für Otto Mayer von 1916), in: Ders., Ausgewählte Schriften und Reden, Zürich 1941, S. 138-162. • »Politik als Wissenschaft« (1917), in: Ders., Ausgewählte Schriften und Reden, Zürich 1941, S. 181196. - "Schweizerische und deutsche Staatsauffassung" (1929), in: Ders., Ausgewählte Schriften und Reden, Zürich 1941, S. 235249. - »Tradition, Dogma, Entwicklung als aufbauende Kräfte der schweizerischen Demokratie« (1933), in: Ders., Ausgewählte Schriften und Reden, Zürich 1941, S. 288-302. - Institutionen des Deutschen



F O R S T H O F F, Ernst: Lehrbuch des Verwaltungsrechts, Bd. I.: Allgemeiner Teil, München 1950. F R A N K E N B E R G , Günter: „Vom Schweigen der Öffentlichrechtler und ihrer Verantwortung, dieses bisweilen zu brechen«, in: Kritische Justiz 27 (1994), S. 354-357. F R O WEIN, Jochen Abr. (Hg.): Die Kontrolldichte bei der gerichtlichen Überprüfung von Handlungen der Verwaltung, Berlin, u. a. 1993. G E N N E R, Susanne: Die Verfügungspflicht der Verwaltungsbehörden: Ein Beitrag zur Geschichte des schweizerischen Verwaltungsrechts, Zürich, u. a. 2013. G I A C O M E T T I, Zaccaria: "Vorwort zum Nachdruck der 8. Auflage«, in: Fritz Fleiner, Institutionen des Deutschen Verwaltungsrechts, Zürich 1939, S. 28-45. - Allgemeine Lehren des rechtsstaatlichen Verwaltungsrechts, Bd. 1. (Bd. 2. nicht erschienen), Zürich 1960. G Ü N T H E R, Frieder: Denken vom Staat her: Die bundesdeutsche Staatsrechtslehre zwischen Dezision und Integration 1949-1970, München 2004. H Ä B E R L E, Peter / K I L I A N , Michael / W O L F F, Heinrich Amadeus (Hg.): Staatsrechtslehrer des 20. Jahrhunderts, Berlin 2015. H A B E R M A S , Jürgen: Faktizität und Geltung, Frankfurt am Main 1998. H A T S C H E K, Julius: Lehrbuch des deutschen und preuBischen Verwaltungsrechts, Leipzig ${ }^{6} 1927$. H E L D - D A A B , Ulla: Das freie Ermessen: Von den vorkonstitutionellen Wurzeln zur positivistischen Auflösung der Ermessenslehre, Berlin 1996. HER R N R I T T, Herrmann: Grundlehren des Verwaltungsrechtes, Tübingen 1921. H E Y E N , Erk Volkmar: Otto Mayer: Studien zu den geistigen Grundlagen seiner Verwaltungsrechtswissenschaft, Berlin 1981. • »Deutschland«, in: Ders. (Hg.), Geschichte der Verwaltungsrechtswissenschaft in Europa: Stand und Probleme der Forschung, Frankfurt am Main 1982, S. 29-50. H U B E R, Hans: »Betrachtungen über die Gesamtsituation des Rechts« (Abschiedsvorlesung von 1970), in: Ders., Ausgewählte Aufsätze, hg. von Kurt Eichenberger, Richard Bäumlin, Jörg P. Müller, Bern 1971, S. 11-26. • "Niedergang des Rechts und Krise des Rechtsstaates" (1953), in: Ders., Ausgewählte Aufsätze, hg. von Kurt Eichenberger, Richard Bäumlin, Jörg P. Müller, Bern 1971, S. 27-56. H U E B E R, Alfons, Otto Mayer: Die »juristische Methode« im Verwaltungsrecht, Berlin 1982. I B L E R, Martin: Rechtspflegender Rechtsschutz im Verwaltungsrecht: Zur Kontrolldichte bei werdenden Behördenentscheidungen vom Preußischen Oberverwaltungsgericht bis zum modernen Gerichtsschutz im Prüfungsrecht, Tübingen 1999. J E L L I N E K, Walter:Gesetz, Gesetzesanwendung und Zweckmässigkeitserwägung, Tübingen 1913. - Verwaltungsrecht, Offenburg: Lehrmittel-Verlag 1948 [unveränderter Nachdruck von ${ }^{31931] . ~ J ~ E ~ S ~ C ~ H, ~ D i e t r i c h: G e s e t z ~ u n d ~ V e r w a l-~}$ tung: Eine Problemstudie zum Wandel des Gesetzmäßigkeitsprinzips, Tübingen 1961. K A H L, Arno / WE BER, Karl: Allgemeines Verwaltungsrecht, Wien 2007. K E M P T E R, Klaus: Die Jellineks (1820-1955): Eine familienbiographische Studie zum deutschjüdischen Bildungsbürgertum, Düsseldorf 1998. K LE Y, Andreas: Von Stampa nach Zürich: Der Staatsrechtler Zaccaria Giacometti, sein Leben und Werk und seine Bergeller Künstlerfamilie, Zürich 2014. • Geschichte des öffentlichen Rechts der Schweiz, Zürich 22015. K Ö L Z, Alfred: "Von der Herkunft des schweizerischen Verwaltungsrechts«, in: Ders., Der Weg der Schweiz zum modernen Bundesstaat, Chur, u. a. 1998, S. 95-116. K O RM A N N, Karl: System der rechtsgeschäftlichen Staatsakte: Verwaltungs- und prozessrechtliche Untersuchungen zum allgemeinen Teil des öffentlichen Rechts, Berlin 1910. K O S E L L E C K, Reinhart: "Standortbindung und Zeitlichkeit. Ein Beitrag zur historiographischen ErschlieBung der geschichtlichen Welt«, in: Ders., Wolfgang J. Mommsen, Jörn Rüsen (Hg.), Objektivität und Parteilichkeit, München S. 17-46. K R A M E R, Ernst A.: Juristische Methodenlehre, München, u. a. ${ }^{42013 . ~ L ~ A ~-~}$ RENZ, Karl / CANARIS, Claus-Wilhelm: Methodenlehre der Rechtswissenschaft, Berlin, u. a. ${ }^{3} 1995$. L A T O U R, Bruno, La fabrique du droit: Une ethnographie du Conseil d'Etat, Paris 2004. LE H N E, Friedrich / L O E BE N STE I N, Edwin / S C H I M ET S C HEK, Bruno 
(Hg.): Die Entwicklung der österreichischen Verwaltungsgerichtsbarkeit: Festschrift zum 100jährigen Bestehen des österreichischen Verwaltungsgerichtshofes, Wien 1976. L E P S I U S, Oliver: Verwaltungsrecht unter dem Common Law, Tübingen 1997. • „Was kann die deutsche Staatsrechtslehre von der amerikanischen Rechtswissenschaft lernen?«, in: Helmuth Schulze-Fielitz (Hg.), Staatsrechtslehre als Wissenschaft, Berlin 2007, S. 319-366. • "Kritik der Dogmatik«, in: Georg Kirchhof, Stefan Magen, Karsten Schneider (Hg.), Was heißt Dogmatik?, Tübingen 2012, S. 39-62. L E R C H E, Peter: »Theodor Maunz (1901-1993)«, in: Peter Häberle, Michael Kilian, Heinrich Amadeus Wolff (Hg.), Staatsrechtslehrer des 20. Jahrhunderts, Berlin 2015, S. 573-578. M A R T I N E K, Michael G.: »Der Rechtskulturschock«, in: Juristische Schulung 24 (1984), S. 92-101. M A S T R O N A R D I, Philippe: Juristisches Denken, Bern 22003. M A U N Z, Theodor: "Das Verwaltungsrecht des nationalsozialistischen Staates«, in: Hans Frank (Hg.), Deutsches Verwaltungsrecht, München 1937, S. 27-48. • Verwaltung, Hamburg 1937. M A U RER， Hartmut: Allgemeines Verwaltungsrecht, München ${ }^{182011 .}$ M A Y E R, Otto: Deutsches Verwaltungsrecht, Bd. I., Leipzig 1895. - Mein Bekenntnis, Halle 1927. - Deutsches Verwaltungsrecht, I. und II. Band, Berlin 2004 [unveränderter Nachdruck von ${ }^{31924] . ~}$ M E I N E L, Florian: „Unser Jahrhundert«, in: Der Staat 54 (2015), S. 231-239. M E R K L , Adolf: Allgemeines Verwaltungsrecht, Wien 1999 [Nachdruck von 1927]. M Ö L L E R S , Christoph: „Historisches Wissen in der Verwaltungsrechtswissenschaft«, in: Eberhard Schmidt-Aßmann, Wolfgang Hoffmann-Riem (Hg.), Methoden der Verwaltungsrechtswissenschaft, Baden-Baden 2004, S. 131-164. M Ü L L E R, Markus, Verwaltungsrecht: Eigenheit und Herkunft, Bern 2006. • - / J EN N I, Christoph: "Die polizeiliche Generalklausel: Ein Institut mit Reformbedarf«, in: Sicherheit \& Recht (2008), S. 4-18. M Ü L L E R, Roger: Verwaltungsrecht als Wissenschaft: Fritz Fleiner 1867-1937, Frankfurt am Main 2006. M U ß G N U G , Reinhard: „§ 8 Zustandekommen des Grundgesetzes und Entstehen der Bundesrepublik Deutschland«, in: Josef Isensee, Paul Kirchhof (Hg.), Handbuch des Staatsrechts der Bundesrepublik Deutschland, Bd. I, Heidelberg ${ }^{3} 2003$. O F T I N G E R, Karl: Vom Handwerkszeug der juristischen Schriftstellerei, Zürich 1944. O L E C H O W S K I, Thomas: Der österreichische Verwaltungsgerichtshof, Wien 2001. P E T E R S, Hans, Zwischen Gestern und Morgen: Betrachtungen zur heutigen Kulturlage, Berlin 1946. R A D B R U C H , Gustav: Einführung in die Rechtswissenschaft, Leipzig 1910. R A P H A E L, Lutz: Geschichtswissenschaft im Zeitalter der Extreme: Theorien, Methoden, Tendenzen von 1900 bis zur Gegenwart, München 22010. RAS C HA U ER, Bernhard:Allgemeines Verwaltungsrecht, Wien 1998. R E I C H, Johannes: Grundsatz der Wirtschaftsfreiheit, Zürich 2011. RE M M E R T, Barbara: Verfassungs- und verwaltungsgeschichtliche Grundlagen des Übermaßverbotes, Heidelberg 1995. R H I N O W, René: "Politische Funktionen des Rechts«, in: Zeitschrift für Schweizerisches Recht 127 (2008) I, S. 181-206. R Ü T H E R S, Bernd: Geschönte Geschichten - Geschonte Biographien, Tübingen 2001. S C H I N D LER, Alfred: »Kirchengeschichte - wozu? «, in: Helge Siemers, Hans-Richard Reuter (Hg.), Theologie als Wissenschaft in der Gesellschaft, Göttingen 1970, S. 140155. S C H I N D L E R, Benjamin: Verwaltungsermessen, Zürich, u. a. 2010. • "100 Jahre Verwaltungsrecht in der Schweiz«, in: Zeitschrift für Schweizerisches Recht 130 (2011) II, S. 331-437. • Staatsrechtslehrer des 20. Jahrhunderts, in: Schweizerisches Zentralblatt für Staats- und Verwaltungsrecht 117 (2016), S. 106-112. S C H M I D T - A B M A N N , Eberhard: Das allgemeine Verwaltungsrecht als Ordnungsidee: Grundlagen und Aufgaben der verwaltungsrechtlichen Systembildung, Heidelberg 22004. S C H Ö N , Wolfgang: "Quellenforscher und Pragmatiker", in: Christoph Engel, Ders., (Hg.), Das Proprium der Rechtswissenschaft, Tübingen 2007, S. 313-321. S C H U L T E, Martin: „Walter Jellinek (1885-1955)«, in: Peter Häberle, Michael Kilian, Heinrich Amadeus Wolff
(Hg.), Staatsrechtslehrer des 20. Jahrhunderts, Berlin 2015, S. 298-311. S C H U L Z E , Gerhard / et al.: Verwaltungsrecht: Lehrbuch, Berlin 1979. S C H U L Z E - F I E L I T Z, Helmut: Staatsrechtslehre als Mikrokosmos: Bausteine zu einer Soziologie und Theorie der Wissenschaft des Öffentlichen Rechts, Tübingen 2013. S C H W E R I N , Claudius Frhr. von: "Geschichte des Verwaltungsrechts und der Verwaltungswissenschaft", in: Hans Frank (Hg.), Deutsches Verwaltungsrecht, München 1937, S. 3-26. S E F E R O V I C, Goran: Das Schweizerische Bundesgericht 1848-1874: Die Bundesgerichtsbarkeit im frühen Bundesstaat, Zürich, u. a. 2010. S E I LER, Hansjörg: Praktische Rechtsanwendung: Was leistet die juristische Methodenlehre?, Bern 2009. S E L B , Walter: "Dogmen und Dogmatik, Dogmengeschichte und Dogmatikgeschichte in der Rechtswissenschaft «, in: Claus-Wilhelm Canaris, Uwe Diederichsen (Hg.), Festschrift für Karl Larenz zum 80. Geburtstag, München 1983, S. 605-614. S T O L L E I S, Michael: "Lehren aus der Geschichte? Zur Auseinandersetzung mit den Thesen Bernd Rüthers (Entartetes Recht)«, in: Rainer Eisfeld, Ingo Müller (Hg.), Gegen Barbarei, Essays Robert M.W. Kempner zu Ehren, Frankfurt am Main 1989, S. 385-395. • "Theodor Maunz - Ein Staatsrechtslehrerleben«, in: Kritische Justiz 26 (1993), S. 393-396. - Rechtsgeschichte schreiben: Rekonstruktion, Erwählung, Fiktion?, Basel 2008. • "Das Zögern beim Blick in den Spiegel: Die deutsche Rechtswissenschaft nach 1933 und nach 1945«, in: Ders., Ausgewählte Aufsätze und Beiträge, hg. von Stefan Ruppert und Milos Vec, Frankfurt am Main 2011, S. 615-634. • "Die Vereinigung der Deutschen Staatsrechtslehrer: Bemerkungen zu ihrer Geschichte«, in: Ders., Ausgewählte Aufsätze und Beiträge, hg. von Stefan Ruppert und Milos Vec, Frankfurt am Main 2011, S. 591-613. • „Zur kritischen Funktion der Rechtsgeschichte«, in: Hagen Hof, Peter Götz von Olenhusen (Hg.), Rechtsgestaltung - Rechtskritik - Konkurrenz von Rechtsordnungen ..., Baden-Baden 2012, S. 212-219. • »Entwicklungsstufen der Verwaltungsrechtswissenschaft «, in: Wolfgang Hoffmann-Riem, Eberhard Schmidt-Aßmann, Andreas Voßkuhle (Hg.), Grundlagen des Verwaltungsrechts, Bd. I, München 22012, S. 65-121. T Ö G E L, Bettina: Die Stadtverwaltung Berns: Der Wandel ihrer Organisation und Aufgaben von 1832 bis zum Beginn der 1920er Jahre, Zürich 2004. T SCHA N N E N, Pierre: Systeme des Allgemeinen Verwaltungsrechts, Bern 2008. VOßKUHLE, Andreas: "Neue Verwaltungsrechtswissenschaft", in: Wolfang Hoffmann-Riem, Eberhard SchmidtAßmann, Andreas Voßkuhle (Hg.), Grundlagen des Verwaltungsrechts, Bd. I, München 22012, S. 1-63. W A H L, Rainer: Herausforderungen und Antworten: Das Öffentliche Recht der letzten fünf Jahrzehnte, Berlin 2006. W H I T E , Hayden: Metahistory: Die historische Einbildungskraft im 19. Jahrhundert, Frankfurt am Main 1991. W O L F F, Hans J. / et.al.: Verwaltungsrecht, Bd. 1, München ${ }^{12} 2007$. W Y S S, Simone: Das subjektive öffentliche Recht als Begriff des Bundesgerichts: Herkunft, Funktion, Berechtigung, Basel 2009. Z S C H O K K E, Heinrich: Des Schweizerlands Geschichte für das Schweizervolk, Aarau ${ }^{3} 1826$. 
1 Ein herzlicher Dank gilt Bardo Fassbender, Markus Müller, Stefan Nellen, Johannes Reich und Anna Rüefli, welche mich mit konstruktiv-kritischen Bemerkungen maßgeblich unterstützt haben.

2 Vgl. Pascale Cancik, "Verwaltungsrechtsgeschichte«, in: Rechtsgeschichte 19 (2011), S. 30-34, hier S. 30; Michael Stolleis, »Entwicklungsstufen der Verwaltungsrechtswissenschaft», in: Wolfgang Hoffmann-Riem, Eberhard Schmidt-Aßmann, Andreas Voßkuhle (Hg.), Grundlagen des Verwaltungsrechts, Bd. I, München 22012, S. 65-121, Rn. 1.

3 Vgl. im Sinne einer Ausnahme Christoph Möllers, "Historisches Wissen in der Verwaltungsrechtswissenschaft«, in: Eberhard SchmidtAßmann, Wolfgang Hoffmann-Riem (Hg.), Methoden der Verwaltungsrechtswissenschaft, Baden-Baden 2004, S. 131-164. Auch für die Rechtsgeschichte Defizite der Selbstreflexion konstatierend: Pio Caroni, Die Einsamkeit des Rechtshistorikers, Basel, u. a. 2005, S. 27.

4 So die Vermutung von Möllers, »Historisches Wissen«, S. 143.

5 Vgl. Lutz Raphael, Geschichtswissenschaft im Zeitalter der Extreme: Theorien, Methoden, Tendenzen von 1900 bis zur Gegenwart, München ${ }^{2} 2010$, S. 50.

6 Pascale Cancik, "Diskussionsvotum an der Jahrestagung der Vereinigung der Deutschen Staatsrechtslehrer 2015«, in: VVDStRL 75 (2016), S. $267 \mathrm{f}$.

7 Zu diesem methodischen Ansatz Hayden White, Metahistory: Die historische Einbildungskraft im 19. Jahrhundert, Frankfurt am Main 1991, S. 9-14. Während White mit Blick auf die Allgemeine Geschichtsschreibung seine Typologie an "archetypischen Erzählformen« der Literatur orientiert (Romanze, Komödie, Tragödie und Satire) folgt die hier vorgenommene Typologie der Verwaltungsrechtsgeschichte theologischen Motiven. Grund hierfür ist die in der älteren Verwaltungsrechtslehre oft anzutreffende Verwendung biblischer Metaphern und die Gemeinsamkeiten zwischen Rechtswissenschaft und Theologie als hermeneutische Disziplinen mit einer Tendenz zur Dogmatisierung. Vgl. die Beiträge in Georg Essen, Nils Jansen (Hg.), Dogmatisierungsprozesse in Recht und Religion, Tübingen 2011.

8 White, Metahistory, S. 9 f. Vgl. auch Benjamin Schindler, Verwaltungsermessen, Zürich, u. a. 2010, Rz. $418 \mathrm{f}$.

9 Zu diesem für die Wissenschaftsgeschichte gerechtfertigten zeitlichen Fokus Cancik, »Verwaltungsrechtsgeschichte», S. 31.

10 Vgl. Philippe Mastronardi, Juristisches Denken, Bern, u. a. ${ }^{22003,}$ Rn. $101 \mathrm{f}$.

11 Vgl. stellvertretend für die reiche Methodenliteratur Ernst A. Kramer, Juristische Methodenlehre, München, u. a. ${ }^{42013, ~ S . ~ 121-152 ; ~}$ Karl Larenz, Claus-Wilhelm Canaris, Methodenlehre der Rechtswissenschaft, Berlin, u. a. ${ }^{31995, ~ S . ~ 149-153 ; ~ H a n s j o ̈ r g ~ S e i l e r, ~ P r a k t i s c h e ~}$ Rechtsanwendung: Was leistet die juristische Methodenlehre?, Bern 2009, S. 43-45.

12 Vgl. etwa den Entscheid des Schweizerischen Bundesgerichts (BGE) 128 I 327 E. 2.3 S. 331 ff.: Das Gericht musste mit Blick auf die Anordnung sicherheitspolizeilicher Maßnahmen eine Bestimmung der Kantonsverfassung aus dem Jahr 1892 auslegen und hierfür die damals verwendete Terminologie in den heutigen Sprachgebrauch "übersetzen«.

13 Karl Oftinger, Vom Handwerkszeug der juristischen Schriftstellerei, Zürich 1944, S. $37 \mathrm{f}$.

14 Stolleis, "Entwicklungsstufen der Verwaltungsrechtswissenschaft", Rn. 60. Heyen bezeichnet das Werk als "Angelpunkt der wissenschaftsgeschichtlichen Periodisierung«: Erk Volkmar Heyen, "Deutschland«, in: ders. (Hg.), Geschichte der Verwaltungsrechtswissenschaft in Europa: Stand und Probleme der Forschung, Frankfurt am Main 1982, S. 29-50, hier S. 29.

15 Otto Mayer, Deutsches Verwaltungsrecht, I. und II. Band, Berlin 2004 [unveränderter Nachdruck von ${ }^{31924]}$, Bd. I, S. 25-63.
16 Mayer, Deutsches Verwaltungsrecht, Bd. I, S. 25 f.

17 Fritz Fleiner, Institutionen des Deutschen Verwaltungsrechts, Aalen 1995 [unveränderter Nachdruck von ${ }^{81928], ~ S . ~ 28-45 . ~}$

18 Fleiner, Institutionen, S. 28 (Hervorhebung im Original).

19 Fritz Fleiner, Schweizerisches Bundesstaatsrecht, Tübingen 1923, Vorwort.

20 Vgl. auch Fleiner, Schweizerisches Bundesstaatsrecht, Vorwort: »In langsamen Wandlungen ist dieses Recht entstanden; eine Schicht hat sich über die andere gelegt."

21 Vgl. statt vieler Dirk Ehlers, »Otto Mayer (1846-1924)«, in: Peter Häberle, Michael Kilian, Heinrich Amadeus Wolff (Hg.), Staatsrechtslehrer des 20. Jahrhunderts, Berlin, u. a. 2015, S. 46-56, hier S. 48. Eingehend mit dem Herkunftsmilieu Mayers befasst hat sich Erk Volkmar Heyen, Otto Mayer: Studien zu den geistigen Grundlagen seiner Verwaltungsrechtswissenschaft, Berlin 1981.

22 Vgl. statt vieler Giovanni Biaggini, »Fritz Fleiner (1867-1937)«, in: Häberle/Kilian/Wolff, Staatsrechtslehrer, S. 110-126, hier S. 112 f.; Roger Müller, Verwaltungsrecht als Wissenschaft: Fritz Fleiner 1867-1937, Frankfurt am Main 2006, S. 5 f.

23 Vgl. Heyen, Otto Mayer, S. 29.

24 Heinrich Zschokke, Des Schweizerlands Geschichte für das Schweizervolk, Aarau ${ }^{3} 1826$, S. 3.

25 Alfons Hueber, Otto Mayer: Die »juristische Methode« im Verwaltungsrecht, Berlin 1982, S. $154 \mathrm{f}$.

26 Fleiner betont denn auch die Pionierrolle Otto Mayers und verweist darauf, dass dieser mit seinen Werken »die Bahn gebrochen« habe (Fleiner, Institutionen, S. 44).

27 Zum Bemühen Fleiners, »die traditionsvermittelnde Identität eines homogenen Kontinuums zu suggerieren« Müller, Verwaltungsrecht als Wissenschaft, S. 78. Auch bei Fleiner klingen aber teilweise fortschrittsgläubige Töne an. Vgl. etwa Fleiner, Institutionen, S. 42.

28 Fritz Fleiner, »Tradition, Dogma, Entwicklung als aufbauende Kräfte der schweizerischen Demokratie« (1933), in: Ders., Ausgewählte Schriften und Reden, Zürich 1941, S. 288-302, hier S. 291, S. 302.

29 Fritz Fleiner, »Politik als Wissenschaft» (1917), in: Ders., Ausgewählte Schriften und Reden, Zürich 1941, S. 181-196, hier S. 196.

30 Exemplarisch etwa Herrmann Herrnritt, Grundlehren des Verwaltungsrechtes, Tübingen 1921, S. 36.

31 Mayer, Deutsches Verwaltungsrecht, Bd. I, S. 13, Fn. 2.

32 Vgl. Martin Schulte, "Walter Jellinek (1885-1955)«, in: Häberle/Kilian/Wolff, Staatsrechtslehrer, S. 298-311. Lesenswert ist die familienbiografische Einordnung bei Klaus Kempter, Die Jellineks (18201955): Eine familienbiographische Studie zum deutschjüdischen Bildungsbürgertum, Düsseldorf 1998, S. 412-550.

33 Walter Jellinek, Verwaltungsrecht, Offenburg 1948 [unveränderter Nachdruck von ${ }^{31931]}$.

34 Zur nach 1945 verstärkten Tendenz der Enthistorisierung der Disziplin vgl. hinten, "Geschichte als Kontrastfolie».

35 Ernst Forsthoff, Lehrbuch des Verwaltungsrechts, Bd. I.: Allgemeiner Teil, München 1950, S. 15.

36 Ebenda, S. 15, Fn. 1.

37 Ebenda, S. 263.

38 Hierzu Möllers, »Historisches Wissen«, S. 150.

39 Otto Mayer, Mein Bekenntnis, Halle 1927, S. 51.

40 Ebenda, S. 51: „Bei der fortschreitenden Entwicklung von unteren zu höheren Stufen, wie sie in der Welt sich vollzieht, geht es selbstverständlich nicht ab ohne ein schmerzhaftes Sichdurchdringen des Neuen und Absterben des Alten."

41 Mayer, Deutsches Verwaltungsrecht, Bd. I., S. 135.

42 Forsthoff, Lehrbuch des Verwaltungsrechts, S. 44.

43 Mayer, Deutsches Verwaltungsrecht, Bd. I, S. 58. 
44 Ebenda, S. 62.

45 Vgl. Ebenda, S. 64-73 (»§ 6, Die Herrschaft des Gesetzes«), S. 92-103 (§ 9, »Der Verwaltungsakt«), S. 122-199 (»Dritter Abschnitt: Der Rechtsschutz in Verwaltungssachen«). Vgl. auch dasselbe Kernprogramm bei Jellinek, Verwaltungsrecht, S. 96.

46 Vgl. Heyen, Otto Mayer, S. 165 f.

47 Mayer, Mein Bekenntnis, S. 51: »Ihre geistige Entwicklung hat sie [die Menschheit] hoch über das Tier gehoben, aber nach der einen Seite hängt sie immer noch stark mit der Art dieser geringeren Welt zusammen. Der nächste Schritt im großen Gang aufwärts führt sie nicht zu dem kindischen Zerrbild des Übermenschen, sondern richtig über das Menschenmaß hinaus: näher zu Gott. « Zu Mayers Verhältnis zur theologisch-eschatologischen Literatur, Ebenda, S. 55. Skeptisch zur Wechselwirkung zwischen Mayers religiösem Denken und seinem Geschichtsbild Heyen, Otto Mayer, S. 166.

48 Ähnlich Herrnritt, Grundlehren, S. 37. Vgl. hierzu Heyen, Otto Mayer, S. 125.

49 Dieser Wandel zeigte sich in der Auflage von 1928 etwa in der stärkeren Berücksichtigung der auf Leistungserbringung ausgerichteten Verwaltung und der damit verbundenen "Flucht ins Privatrecht" (Fleiner, Institutionen, S. 326) sowie in einem konsequenten Begriffswechsel vom »Untertanen« zum »Bürger« (vgl. exemplarisch a. a. O., S. 165)

50 Fleiner, Institutionen, S. 40. Fleiner hat diese Metapher in verschiedenen Schriften immer wieder verwendet. Vgl. die zahlreichen Hinweise bei Müller, Verwaltungsrecht als Wissenschaft, S. 233 Fn. 273, S. 302 Fn. 181.

51 Die Metapher wird in der Literatur meist auf Gustav Radbruch zurückgeführt (Einführung in die Rechtswissenschaft, Leipzig 1910, S. 91), lässt sich aber bereits im 19. Jahrhundert nachweisen (vgl. mit Hinweisen Schindler, Verwaltungsermessen, Rz. 87).

52 Zaccaria Giacometti, Allgemeine Lehren des rechtsstaatlichen Verwaltungsrechts, Bd. 1. (Bd. 2. nicht erschienen), Zürich 1960.

53 Giacometti, Allgemeine Lehren, S. 470. Vgl. auch die Aussage auf S. 490, wonach »diese Vollendung des Rechtsstaates noch nicht erreicht« sei. Fleiner äußerte sich in einer Vorlesung von 1922 dahin, dass die Schweiz in Bezug auf die Verwaltungsgerichtsbarkeit »der rückständigste Staat in Europa« sei (Nachweis bei Müller, Verwaltungsrecht als Wissenschaft, S. 298 [Anmerkung FN 169]).

54 Rezension von Fritz Stier-Somlo aus dem Jahr 1912 (hier zitiert nach Müller, Verwaltungsrecht als Wissenschaft, S. 177).

55 Müller, Verwaltungsrecht als Wissenschaft, S. 177.

56 Fritz Fleiner, "Beamtenstaat und Volksstaat" (aus der Festgabe für Otto Mayer von 1916), in: Ders., Ausgewählte Schriften und Reden, Zürich 1941, S. 138-162, hier S. 162.

57 Vgl. Schindler, Verwaltungsermessen, Rz. 121.

58 Benjamin Schindler, "100 Jahre Verwaltungsrecht in der Schweiz", in: Zeitschrift für Schweizerisches Recht 130 (2011) II, S. 331-437, hier S. 419.

59 Fleiner, Institutionen, Vorwort zur ersten Auflage von 1911 (in allen späteren Auflagen mit abgedruckt).

60 Jellinek, Verwaltungsrecht, S. 96.

61 Ebenda, S. 97.

62 Vgl. Jürgen Habermas, Faktizität und Geltung, Frankfurt am Main 1998 , S. 308

63 Vgl. Schindler, Verwaltungsermessen, Rz. 83.

64 Fleiner, Schweizerisches Bundesstaatsrecht, S. 763: "Wie sehr tut dieses rast- und ziellose `Gesetzgebern fachheit und Klarheit der Gesetze und der Rechtssicherheit Eintrag!«.

65 Vgl. insb. mit Blick auf die schweizerische Literatur Johannes Reich, Grundsatz der Wirtschaftsfreiheit, Zürich 2011, Rz. 162-166, 487-490.
66 Vgl. neuerdings für den angelsächsischen Raum Niall Ferguson, The Great Degeneration: How Institutions Decay and Economies Die, London 2012, S. 78-110.

$67 \mathrm{Zu}$ dieser Metapher Reich, Grundsatz der Wirtschaftsfreiheit, Rz. 489.

68 Hans Huber, "Niedergang des Rechts und Krise des Rechtsstaates« (1953), in: Ders., Ausgewählte Aufsätze, hg. von Kurt Eichenberger, Richard Bäumlin, Jörg P. Müller, Bern 1971, S. 27-56.

69 Hans Huber, "Betrachtungen über die Gesamtsituation des Rechts" (Abschiedsvorlesung von 1970), in: Ders., Ausgewählte Aufsätze, S. 11-26, hier S. 26.

70 Adolf Merkl, Allgemeines Verwaltungsrecht, Wien 1999 [Nachdruck von 1927].

71 Merkl, Allgemeines Verwaltungsrecht, S. 66-68. Vgl. auch S. 342

72 Ebenda, S. 63.

73 Ebenda, Vorwort, S. XIV.

74 Vgl. Michael Stolleis, "Das Zögern beim Blick in den Spiegel: Die deutsche Rechtswissenschaft nach 1933 und nach 1945«, in: Ders., Ausgewählte Aufsätze und Beiträge, hg. von Stefan Ruppert und Miloš Vec, Frankfurt am Main 2011, S. 615-634, hier S. 629.

75 Vgl. Horst Dreier, "Merkls Verwaltungsrechtslehre und die deutsche Dogmatik des Verwaltungsrechts«, in: Robert Walter (Hg.), Adolf J. Merkl: Werk und Wirksamkeit, Wien 1990, S. 55-88; Möllers, »Historisches Wissen«, S. 157.

76 Vgl. Frieder Günther, Denken vom Staat her: Die bundesdeutsche Staatsrechtslehre zwischen Dezision und Integration 1949-1970, München 2004, S. 112-191; Michael Stolleis, »Die Vereinigung der Deutschen Staatsrechtslehrer: Bemerkungen zu ihrer Geschichte«, in: Ders., Ausgewählte Aufsätze und Beiträge, S. 591-613, hier S. $612 \mathrm{f}$.

77 N. N. [= Helmut Schulze-Fielitz], Die deutschen Staatsrechtslehrer der Gegenwart und ihre akademische Herkunft, Baden-Baden: Nomos 1999 (vier großformatige Blätter). Hierzu den »relativierenden Kommentar« von Helmut Schulze-Fielitz, Staatsrechtslehre als Mikrokosmos: Bausteine zu einer Soziologie und Theorie der Wissenschaft des Öffentlichen Rechts, Tübingen 2013, S. 453-469.

78 Vgl. die Widmung in Karl Kormann, System der rechtsgeschäftlichen Staatsakte: Verwaltungs- und prozessrechtliche Untersuchungen zum allgemeinen Teil des öffentlichen Rechts, Berlin 1910: „Dem Meister deutscher Verwaltungsrechtswissenschaft Otto Mayer in dankbarer Verehrung«. Vgl. auch die Widmung (für Otto Mayer) in Walter Jellinek, Gesetz, Gesetzesanwendung und Zweckmässigkeitserwägung, Tübingen 1913.

79 Vgl. aus der aktuellen Literatur: Hartmut Maurer, Allgemeines Verwaltungsrecht, München ${ }^{18} 2011$, S. 19.

80 Jellinek, Verwaltungsrecht, S. 104-107. Immerhin erwähnt Jellinek, dass nicht "alle nach 1895 erschienenen Schriften von Otto Mayer beeinflußt« wurden (S. 107) - womit er aber unterstreicht, dass alle anderen Werke in der Nachfolge Mayers verfasst wurden.

81 Jellinek, Verwaltungsrecht, S. 106.

82 Vgl. exemplarisch die Werke von Günther, Kempter, Stolleis sowie Andreas Kley, Von Stampa nach Zürich: Der Staatsrechtler Zaccaria Giacometti, sein Leben und Werk und seine Bergeller Künstlerfamilie, Zürich 2014.

83 Peter Häberle, Michael Kilian, Heinrich Amadeus Wolff (Hg.), Staatsrechtslehrer des 20. Jahrhunderts, Berlin 2015.

84 Häberle/Kilian/Wolff (Hg.), Staatsrechtslehrer des 20. Jahrhunderts, S. VII.

85 Vgl. hierzu die Rezensionen von Florian Meinel, "Unser Jahrhundert«, in: Der Staat 54 (2015), S. 231-239 und Benjamin Schindler, "Staatsrechtslehrer des 20. Jahrhunderts«, in: Schweizerisches Zen- 
tralblatt für Staats- und Verwaltungsrecht 117 (2016), S. 106-112.

86 Vgl. exemplarisch zu dieser Phase in Maunz' Leben: Michael Stolleis, "Theodor Maunz - Ein Staatsrechtslehrerleben«, in: Kritische Justiz 26 (1993), S. 393-396.

87 Die Verleihung des Ehrentitels »Ehrwürdiger Diener Gottes« ist ein Verfahrensschritt im Seligsprechungsprozess der römisch-katholischen Kirche.

88 Peter Lerche, "Theodor Maunz (1901-1993)«, in: Häberle/Kilian/ Wolff (Hg.), Staatsrechtslehrer des 20. Jahrhunderts, S. 573-578, hier S. $574 \mathrm{f}$.

89 Das Werk erschien 1919 unter dem an Fleiner anknüpfenden Titel »Institutionen des deutschen und preußischen Verwaltungsrechts«. Ab der 2. Auflage (1922) erfolgte dann der Wechsel zum hier zitierten Titel.

90 Julius Hatschek, Lehrbuch des deutschen und preußischen Verwaltungsrechts, Leipzig ${ }^{6} 1927$, S. 1.

91 Vgl. die Hinweise in Fußnote 61: Im Nachtrag zur 3. Aufl. von 1934 ist von den kämpferischen Aussagen aus dem Jahr 1931 nicht mehr viel zu spüren. Vielmehr sind die Ausführungen vom Bemühen geprägt, sich mit den neuen politischen Verhältnissen zu arrangieren. Das Konzept des Rechtsstaats wird zwar nicht aufgegeben. Der liberale Rechtsstaat weicht nun aber dem »staatsbetonten Rechtsstaat" (a. a. O., S. 9).

92 Theodor Maunz, Verwaltung, Hamburg 1937, S. 30.

93 Ebenda, S. $16 \mathrm{f}$.

94 Theodor Maunz, "Das Verwaltungsrecht des nationalsozialistischen Staates«, in: Hans Frank (Hg.), Deutsches Verwaltungsrecht, München 1937, S. 27-48, hier S. 47.

95 Maunz, Verwaltung, S. 17

96 Claudius Frhr. von Schwerin, „Geschichte des Verwaltungsrechts und der Verwaltungswissenschaft «, in: Hans Frank (Hg.), Deutsches Verwaltungsrecht, S. 3-26, hier S. 6 f.

97 Ebenda, S. 7

98 Maunz, Verwaltung, S. 16-19.

99 Der erste Abschnitt seines Werks trägt den Titel »Das Vorstellungsbild des bürgerlich-rechtsstaatlichen Verwaltungsrechts und seine Überwindung«: Ebenda, S. 30-73.

$100 \mathrm{Vgl}$. Ebenda.

101 Die DDR definierte sich als »Antipode zum bürgerlichen Staat», weshalb auch dessen Verwaltungsrecht als Teil seiner »diktatorischen Herrschaftsmethoden « verstanden wurde und Otto Mayer zur Zielscheibe der Kritik wurde: Gerhard Schulze, et. al., Verwaltungsrecht: Lehrbuch, Berlin 1979, S. 32, S. 59-61.

102 Zaccaria Giacometti, „Vorwort zum Nachdruck der 8. Auflage«, in: Fritz Fleiner, Institutionen des Deutschen Verwaltungsrechts, Zürich 1939, S. 28-45.

103 Christian Bumke, „Die Entwicklung der verwaltungsrechtswissenschaftlichen Methodik in der Bundesrepublik Deutschland «, in: Eberhard Schmidt-Aßmann, Wolfgang Hoffmann-Riem (Hg.), Methoden der Verwaltungsrechtswissenschaft, Baden-Baden 2004, S. 73-130, hier S. 93

104 Vgl. Ebenda, S. 91-93 sowie vorne, Abschnitt „Geschichte als Baum der Erkenntnis«, Fokussierung auf juristische Fragestellungen und Epochenbildung.

$105 \mathrm{Vgl}$. etwa den programmatischen Grundsatzbeitrag zur dreibändigen Monumentalreihe: Andreas Voßkuhle, „Neue Verwaltungsrechtswissenschaft", in: Wolfang Hoffmann-Riem, Eberhard Schmidt-Aßmann, Andreas Voßkuhle (Hg.), Grundlagen des Verwaltungsrechts, Bd. I, München ${ }^{2} 2012$, S. 1-63. Aus kritischer Distanz (mit zahlreichen weiteren Hinweisen): Christoph Engel, »Herrschaftsausübung bei offener Wirklichkeitsdefinition«, in: Ders., Wolfgang Schön (Hg.), Das Proprium der Rechtswissenschaft, Tübingen 2007, S. 205-240;
Rainer Wahl, Herausforderungen und Antworten: Das Öffentliche Recht der letzten fünf Jahrzehnte, Berlin 2006, S. 87-94. 106 Voßkuhle, »Neue Verwaltungsrechtswissenschaft«, S. 5. 107 Vgl. Ebenda, S. 21-41; Wahl, Herausforderungen, S. 89.

108 Vgl. Schindler, Verwaltungsermessen, Rz. 320; Wahl, Herausforderungen, S. 87-94.

109 Vgl. Wahl, Herausforderungen, S. 93.

110 Vgl. Mayer, Deutsches Verwaltungsrecht, Bd. I, S. 20.

$111 \mathrm{Vgl}$. Wahl, Herausforderungen, S. $93 \mathrm{f}$.

112 Ausnahmen bilden diesbezüglich in Deutschland die Werke von Maurer, Allgemeines Verwaltungsrecht, S. 13-38; und Hans J. Wolff, et al., Verwaltungsrecht, Bd. 1, München: ${ }^{12} 2007$, S. 67-120 (das letztgenannte umfangreiche und dreibändige Werk ist indes nur noch bedingt für den universitären Lehrbetrieb geeignet). Diese Tendenz konstatierend: Möllers, »Historisches Wissen«, S. 144-147.

113 Die schweizerische Verwaltungsrechtsliteratur kommt weitestgehend ohne historische Betrachtungen aus. Ausnahme bildet Markus Müller, Verwaltungsrecht: Eigenheit und Herkunft, Bern 2006, der auf den fehlenden Bezug zur Geschichte hinweist (S. 106).

114 Vgl. die historischen Einleitungen in Ludwig K. Adamovich, et al., Österreichisches Staatsrecht, Bd. 4: Allgemeine Lehren des Verwaltungsrechts, Wien 2009, S. 3-28; Walter Antoniolli, Friedrich Koja, Allgemeines Verwaltungsrecht, Wien ${ }^{3} 1996$, S. 119-142; Arno Kahl, Karl Weber, Allgemeines Verwaltungsrecht, Wien 2007, S. 34-41, S. 50 f.; Bernhard Raschauer, Allgemeines Verwaltungsrecht, Wien 1998 enthält in der ersten Auflage eine historische Einleitung (S. 12-21), die dann aber seit der 2. Auflage (2003; aktuelle 4. Auflage von 2013) fehlt.

$115 \mathrm{Vgl}$. Schindler, Verwaltungsermessen, Rn. $93 \mathrm{f}$.

116 Möllers, »Historisches Wissen«, S. $148 \mathrm{f}$.

117 So Reinhard Mußgnug, "§ 8 Zustandekommen des Grundgesetzes und Entstehen der Bundesrepublik Deutschland «, in: Josef Isensee, Paul Kirchhof (Hg.), Handbuch des Staatsrechts der Bundesrepublik Deutschland, Bd. I, Heidelberg ${ }^{3} 2003$, Rn. 51.

118 Während Walter Jellinek kurz nach Kriegsende noch eine "Entsühnung« gefordert hatte, befürwortete er später eine »nicht-symmetrische Diskretion«, um den »Belasteten« die Integration im neuen, demokratischen Staatswesen zu ermöglichen (Kempter, Die Jellineks, S. 537). Jellinek kann zwar aufgrund seiner rassisch motivierten Entfernung aus der Universität Heidelberg als >Opfer des Nationalsozialismus gelten; seine Schriften aus den 1930er-Jahren (vgl. Fußnote 91) dürften aber auch bei ihm ein Bedürfnis nach Diskretion gefördert haben.

119 Bernd Rüthers, Geschönte Geschichten - Geschonte Biographien, Tübingen 2001, S. 22; vgl. Günter Frankenberg, "Vom Schweigen der Öffentlichrechtler und ihrer Verantwortung, dieses bisweilen zu brechen«, in: Kritische Justiz 27 (1994), S. 354-357; Günther, Denken vom Staat her, S. 66-77, S. 117; Michael Stolleis, „Entwicklungsstufen der Verwaltungsrechtswissenschaft«, Rn. 92-94.

120 Vgl. den Beitrag Peter Lerches über Theodor Maunz aus dem Jahr 2015 (Fußnote 88).

121 So die Formulierung von Edgar Tatarin-Tarnheyden in einem Brief aus dem Jahr 1954, worin er sich dafür bedankt, dass er trotz seiner antisemitischen Vergangenheit wieder Aufnahme im Kreis der Vereinigung der Deutschen Staatsrechtslehrer gefunden hat (zitiert nach Günther, Denken vom Staat her, S. 70).

122 Hans Peters, Zwischen Gestern und Morgen: Betrachtungen zur heutigen Kulturlage, Berlin 1946, S. 15 (Hervorhebungen im Original).

123 Mayer, Deutsches Verwaltungsrecht, Bd. I, Vorwort zur 3. Auflage.

124 Forsthoff, Lehrbuch des Verwaltungsrechts, Vorwort zur ersten Auflage: „Im Ablauf der mit dem Untergang der Monarchie beginnenden, heute noch nicht überwundenen Staatskrise zeichnet sich 
hinter den wechselnden Verfassungsszenerien eine konstante Entwicklung ab."

125 Zum traditionell großen Einfluss der Politik auf die Verwaltung in der Schweiz vgl. Fritz Fleiner, "Schweizerische und deutsche Staatsauffassung« (1929), in: Ders., Ausgewählte Schriften und Reden, Zürich 1941, S. 235-249, hier S. 242. Aus jüngerer Zeit vgl. René Rhinow, »Politische Funktionen des Rechts«, in: Zeitschrift für Schweizerisches Recht 127 (2008) I, S. 181-206.

126 Kormann, System, S. 2; vgl. auch Mayer, Deutsches Verwaltungsrecht, Bd. I, S. 21.

127 Den Systemgedanken besonders betonend etwa Pierre Tschannen, Systeme des Allgemeinen Verwaltungsrechts, Bern 2008. Aus der älteren Literatur Winfried Brohm, »Die Dogmatik des Verwaltungsrechts vor den Gegenwartsaufgaben der Verwaltung «, in: VVDStRL 30 (1971), Berlin, New York 1972, S. 245-364, hier S. 246, S. 248 f. Hierzu (mit weiteren Nachweisen) Oliver Lepsius, „Kritik der Dogmatik«, in: Georg Kirchhof, Stefan Magen, Karsten Schneider (Hg.), Was heißt Dogmatik?, Tübingen 2012, S. 39-62, hier S. 40; Müller, Verwaltungsrecht als Wissenschaft, S. 210-248; Schindler, "100 Jahre«, S. 382; Walter Selb, „Dogmen und Dogmatik, Dogmengeschichte und Dogmatikgeschichte in der Rechtswissenschaft«, in: ClausWilhelm Canaris, Uwe Diederichsen (Hg.), Festschrift für Karl Larenz zum 80. Geburtstag, München 1983, S. 605-614, hier S. 611.

128 Eberhard Schmidt-Aßmann, Das allgemeine Verwaltungsrecht als Ordnungsidee: Grundlagen und Aufgaben der verwaltungsrechtlichen Systembildung, Heidelberg ${ }^{2} 2004$, S. 1-41; Voßkuhle, "Neue Verwaltungsrechtswissenschaft", S. $40 \mathrm{f}$.

129 Tschannen, Systeme, S. 1.

130 So Brohm, »Die Dogmatik des Verwaltungsrechts vor den Gegenwartsaufgaben der Verwaltung «, S. 248; vgl. etwa Thomas von Danwitz, Europäisches Verwaltungsrecht, Berlin 2008, S. 158 f., der auf das "Rechtsquellenwirrwarr" im europäischen Verwaltungsrecht verweist und fordert, dieses "in eine systematische Ordnung" zu bringen und »die verschiedenen Rechtssätze einander so zuzuordnen, dass ein widerspruchsfreies, kohärentes Ganzes entsteht«.

131 Vgl. Lepsius, »Kritik der Dogmatik«, S. 55 f.

132 Zur Abneigung gegenüber dem Systemdenken, insb. für die USA, Oliver Lepsius, Verwaltungsrecht unter dem Common Law, Tübingen 1997, S. 302, S. 306; Ders., "Was kann die deutsche Staatsrechtslehre von der amerikanischen Rechtswissenschaft lernen?«, in: Helmuth Schulze-Fielitz (Hg.), Staatsrechtslehre als Wissenschaft, Berlin 2007, S. 319-366, hier S. 326-330, S. 335-337.

133 Besonders in der deutschen Rechtswissenschaft ist die Ansicht weit verbreitet, dass eine begrifflich und systematisch betriebene Rechtswissenschaft der kasuistischen Rechtstradition des Common Law überlegen sei. Vgl. Jellinek, Verwaltungsrecht, S. 112, der vom englischen Verwaltungsrecht als »verkümmertem Wissenszweig" spricht, sowie aus jüngerer Zeit das Votum von Matthias Herdegen, in: Jochen Abr. Frowein (Hg.), Die Kontrolldichte bei der gerichtlichen Überprüfung von Handlungen der Verwaltung, Berlin, u. a. 1993, S. 303, der von einem »Nachholbedarf an Systematisierung« spricht. Vgl. auch Lepsius, Verwaltungsrecht unter dem Common Law, der in der mangelnden systematischen Durchdringung des amerikanischen Verwaltungsrechts ein "Substanzproblem« erblickt (S. 303). Kritisch zu diesem »Chauvinismus« Michael G. Martinek, »Der Rechtskulturschock«, in: Juristische Schulung 24 (1984), S. 92-101, hier S. 99.

$134 \mathrm{Vgl}$. Möllers, »Historisches Wissen«, S. $146 \mathrm{f}$.

$135 \mathrm{Vgl}$. Armin von Bogdandy, „Verwaltungsrecht im europäischen Rechtsraum - Perspektiven einer Disziplin«, in: Ders., Sabino Cassese, Peter M. Huber (Hg.), Handbuch Ius Publicum Europaeum, Bd.
IV, Heidelberg 2011, S. 3-36, hier S. 31; Lepsius, "Was kann die deutsche Staatsrechtslehre von der amerikanischen Rechtswissenschaft lernen?« S. 337 f.; Martinek, »Der Rechtskulturschock«, S. 100.

136 Vgl. exemplarisch den Beitrag von Stolleis, »Entwicklungsstufen der Verwaltungsrechtswissenschaft«.

137 Vgl. etwa das Werk von Wolff, Bachof, Stober, Kluth (Fußnote 112), welches zwar in der Reihe »uristische Kurz-Lehrbücher « erscheint, mit drei Bänden zu mehreren hundert Seiten diesem Anspruch aber nur noch schwerlich gerecht wird. Die umgekehrte Entwicklung eines Werks ist eher selten zu beobachten. Ins Auge sticht immerhin das Werk von Raschauer (Fußnote 114), welches nach der ersten Auflage deutlich gestrafft wurde: Neben einer stärkeren Fokussierung auf aktuelle Rechtsentwicklungen entfiel die geschichtliche Einleitung, und auf Fußnoten wurde verzichtet.

138 Michael Stolleis, "Zur kritischen Funktion der Rechtsgeschichte», in: Hagen Hof, Peter Götz von Olenhusen (Hg.), Rechtsgestaltung Rechtskritik - Konkurrenz von Rechtsordnungen ..., Baden-Baden 2012, S. 212-219, hier S. 216.

139 Vgl. Cancik, »Verwaltungsrechtsgeschichte«, S. 34; Caroni, Die Einsamkeit des Rechtshistorikers, S.12. Aus kirchengeschichtlich-theologischer Perspektive vgl. Alfred Schindler, "Kirchengeschichte - wozu?», in: Helge Siemers, Hans-Richard Reuter (Hg.), Theologie als Wissenschaft in der Gesellschaft, Göttingen 1970, S. 140-155, hier S. 153.

140 Michael Stolleis, »Lehren aus der Geschichte? Zur Auseinandersetzung mit den Thesen Bernd Rüthers (Entartetes Recht)«, in: Rainer Eisfeld, Ingo Müller (Hg.), Gegen Barbarei, Essays Robert M.W. Kempner zu Ehren, Frankfurt am Main 1989, S. 385-395, hier S. 385.

141 Hierzu vorne, zu Beginn von Abschnitt "Geschichte als Baum der Erkenntnis«.

142 Fleiner, Institutionen, Vorwort zur ersten Auflage von 1911.

143 Vgl. Jellinek, Verwaltungsrecht, S. 106. Zur Verwendung von architektonischen Metaphern in der Sprache des Verwaltungsrechts vgl. m. w. Hw. Schindler, "100 Jahre», S. 384-386.

144 Vgl. Martinek, »Der Rechtskulturschock«, S. 100.

145 Schindler, »100 Jahre«, S. $388 \mathrm{f}$.

146 Vgl. Bodo Dennewitz, Die Systeme des Verwaltungsrechts, Hamburg 1948, S. 11.

147 Vgl. Jörg Baberowski, Der Sinn der Geschichte: Geschichtstheorien von Hegel bis Foucault, München 2005, S. 207; Michael Stolleis, Rechtsgeschichte schreiben: Rekonstruktion, Erwählung, Fiktion?, Basel 2008, S. 27.

148 Vgl. Möllers, »Historisches Wissen«, S. 152-154.

$149 \mathrm{Zu}$ diesem transdisziplinären Dialog in der Verwaltungsgeschichte vgl. Gilbert Coutaz, et al., »Was soll und kann Verwaltungsgeschichte?«, in: traverse 3 (2011), S. 160-170.

150 Vgl. Stolleis, Rechtsgeschichte schreiben, S. 5.

151 Sabino Cassesse (1995), zitiert nach Müller, Verwaltungsrecht: Eigenheit und Herkunft, S. 29. Diese These hat in der Schweiz viel Anklang gefunden. Vgl. Alfred Kölz, "Von der Herkunft des schweizerischen Verwaltungsrechts«, in: Ders., Der Weg der Schweiz zum modernen Bundesstaat, Chur, u. a. 1998, S. 95-116; Markus Müller, a. a. O; vgl. die Kritik an dieser zu einseitigen Rezeptionsthese bei Andreas Kley, Geschichte des öffentlichen Rechts der Schweiz, Zürich 22015, S. 89; Schindler, »100 Jahre«, S. 389-391.

152 Vgl. Baberowski, Der Sinn der Geschichte, S. 207 f.; Möllers, »Historisches Wissen«, S. 140 f.; Stolleis, Rechtsgeschichte Schreiben, S. 40-43. 153 Vgl. Cancik, "Diskussionsvotum«, S. 267 f.

154 Vgl. Baberowski, Der Sinn der Geschichte, S. 213; Wolfgang Schön, "Quellenforscher und Pragmatiker«, in: Christoph Engel, Ders. (Hg.), Das Proprium der Rechtswissenschaft, Tübingen 2007, S. 313-321; Stolleis, Rechtsgeschichte Schreiben, S. 18-21. 
155 Reinhart Koselleck, "Standortbindung und Zeitlichkeit. Ein Beitrag zur historiographischen Erschließung der geschichtlichen Welt», in: Ders., Wolfgang J. Mommsen, Jörn Rüsen (Hg.), Objektivität und Parteilichkeit, München 1977, S. 17-46, hier S. 45 f.

156 Baberowski, Der Sinn der Geschichte, S. 213.

157 Zur Methodenvielfalt in der Geschichtswissenschaft Monika Dommann, David Gugerli, "Geschichtswissenschaft in Begutachtung: Acht Kommentare zur historischen Methode der Gegenwart«, in: traverse 2 (2011), S. 154-164, hier S. 159 f.; für die Rechtswissenschaft vgl. Kramer, Juristische Methodenlehre, S. 55-181.

158 Vgl. Dietrich Jesch, Gesetz und Verwaltung: Eine Problemstudie zum Wandel des Gesetzmäßigkeitsprinzips, Tübingen 1961.

159 Vgl. Barbara Remmert, Verfassungs- und verwaltungsgeschichtliche Grundlagen des Übermaßverbotes, Heidelberg 1995.

160 Vgl. Horst Dreier, Hierarchische Verwaltung im modernen Staat: Genese, aktuelle Bedeutung und funktionelle Grenzen eines Bauprinzips der Exekutive, Tübingen 1991, S. 19-120.

161 Vgl. Susanne Genner, Die Verfügungspflicht der Verwaltungsbehörden: Ein Beitrag zur Geschichte des schweizerischen Verwaltungsrechts, Zürich, u. a. 2013.

162 Vgl. Ulla Held-Daab, Das freie Ermessen: Von den vorkonstitutionellen Wurzeln zur positivistischen Auflösung der Ermessenslehre, Berlin 1996; Schindler, Verwaltungsermessen.

163 Vgl. Simone Wyss, Das subjektive öffentliche Recht als Begriff des Bundesgerichts: Herkunft, Funktion, Berechtigung, Basel 2009.

164 Vgl. hierzu in Ansätzen Hans Boldt, Michael Stolleis, "Geschichte der Polizei in Deutschland», in: Hans Lisken, Erhard Denninger (Hg.), Handbuch des Polizeirechts, München ${ }^{4} 2007$, S. 1-41, hier S. 21; Markus Müller, Christoph Jenni, „Die polizeiliche Generalklausel: Ein Institut mit Reformbedarf«, in: Sicherheit \& Recht 2008, S. 4-18, hier S. 6 f.

$165 \mathrm{Vgl}$. Lepsius, "Was kann die deutsche Staatsrechtslehre von der amerikanischen Rechtswissenschaft lernen?«, S. 365; Möllers, »Historisches Wissen«, S. $161 \mathrm{f}$.

166 Schindler, "Kirchengeschichte - wozu?«, S. 149.

167 In diese Richtung zielt etwa das Werk von Held-Daab (Fußnote 162), welche dem Ermessen als eigenem Forschungsgegenstand schlicht seine Existenzberechtigung abspricht (S. 263).

168 Vgl. Bruno Latour, La fabrique du droit: Une ethnographie du Conseil d'Etat, Paris 2004.

169 Vgl. Christian Ackermann, Die Bedeutung der Rechtsprechung des Preußischen Oberverwaltungsgerichts zum Kommunalrecht für unsere heutige Dogmatik, Baden-Baden 2012; Martin Ibler, Rechtspflegender Rechtsschutz im Verwaltungsrecht: Zur Kontrolldichte bei werdenden Behördenentscheidungen - vom Preußischen Oberverwaltungsgericht bis zum modernen Gerichtsschutz im Prüfungsrecht, Tübingen 1999, S. 202-278.

170 Friedrich Lehne, Edwin Loebenstein, Bruno Schimetschek (Hg.), Die Entwicklung der österreichischen Verwaltungsgerichtsbarkeit: Festschrift zum 100jährigen Bestehen des österreichischen Verwaltungsgerichtshofes, Wien, u. a. 1976; Thomas Olechowski, Der österreichische Verwaltungsgerichtshof, Wien 2001.

$171 \mathrm{Vgl}$. Stephan Aerschmann, Der ideale Richter: Schweizer Bundesrichter in der medialen Öffentlichkeit (1875-2010), Zürich 2014; Goran Seferovic, Das Schweizerische Bundesgericht 1848-1874: Die Bundesgerichtsbarkeit im frühen Bundesstaat, Zürich, u. a. 2010; Christoph Errass, "Zur Geschichte des Bundesgerichts», in: Marcel Alexander Niggli, Peter Uebersax, Hans Wiprächtiger (Hg.), Basler Kommentar zum Bundesgerichtsgesetz, Basel 22011, S. 1-77.

172 Vgl. Fritz Sager, „Was soll und kann Verwaltungsgeschichte?«, in: traverse, Zeitschrift für Geschichte 3, 2011, S. 160-170, hier S. 164.

$173 \mathrm{Vgl}$. exemplarisch die Studie zur Wahrnehmung des Bundesgerichts in der Öffentlichkeit von Aerschmann (Fußnote 171), die wissenschaftsgeschichtliche Arbeit von Günther (Fußnote 76), die Jellineksche Familienbiografie von Kempter (Fußnote 32), die rethnografische`Studie zum Conseil d'Etat von Latour (Fußnote 168) oder die fokussierte Verwaltungsgeschichte von Bettina Tögel, Die Stadtverwaltung Berns: Der Wandel ihrer Organisation und Aufgaben von 1832 bis zum Beginn der 1920er Jahre, Zürich 2004.

174 Vgl. Caroni, Die Einsamkeit des Rechtshistorikers, S. 103.

175 Raphael, Geschichtswissenschaft im Zeitalter der Extreme, S. 50.

176 Adolf Harnack (nach 1914 von Harnack), Sokrates und die alte Kirche. Rede beim Antritt des Rectorates gehalten in der Aula der Königlichen Friedrich-Wilhelms-Universität vom 15. Oktober 1900, Berlin 1900, S. 22 (teilweise wiedergegeben bei Dennewitz, Die Systeme des Verwaltungsrechts, S. 5). 


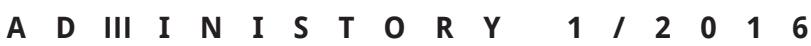

\section{Abstract}

The academic field of administrative law deals above all with the legal framework currently underlying today's public administration. And yet its literature also touches on history, be it that of public administration or administrative law. This article takes a metahistorical approach, investigating the motives behind the field's interest in history and the narrative traditions it follows. Finally, it seeks to answer the question of why scholars of law should play a part in writing administrative history.

\section{About the Author}

Benjamin Schindler, Dr. iur. (Zurich), MJur (Oxford) is Professor of Public Law at the University of St. Gallen (Switzerland). His main interests include administrative and constitutional law from a comparative and historical perspective. In 2012 he was appointed deputy judge of the Constitutional Court of Liechtenstein. 